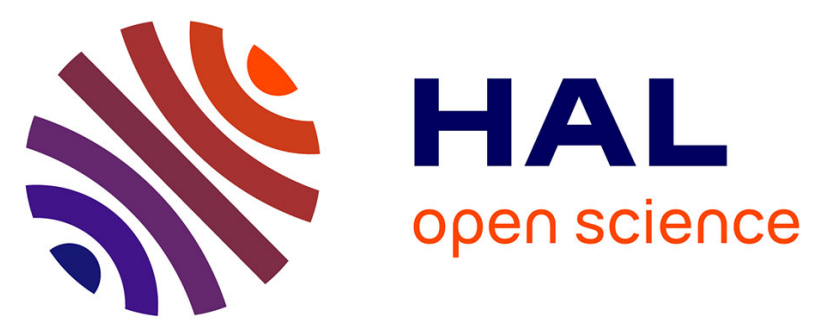

\title{
Non-Markovian transient Casimir-Polder force and population dynamics on excited- and ground-state atoms: Weak- and strong-coupling regimes in generally nonreciprocal environments
}

George W. Hanson, S. Ali Hassani Gangaraj, Mario Silveirinha, Mauro

Antezza, Francesco Monticone

\section{To cite this version:}

George W. Hanson, S. Ali Hassani Gangaraj, Mario Silveirinha, Mauro Antezza, Francesco Monticone. Non-Markovian transient Casimir-Polder force and population dynamics on excited- and ground-state atoms: Weak- and strong-coupling regimes in generally nonreciprocal environments. Physical Review A : Atomic, molecular, and optical physics [1990-2015], 2019, 99, pp.042508. 10.1103/PhysRevA.99.042508 . hal-02103569

\section{HAL Id: hal-02103569 \\ https://hal.science/hal-02103569}

Submitted on 14 Oct 2020

HAL is a multi-disciplinary open access archive for the deposit and dissemination of scientific research documents, whether they are published or not. The documents may come from teaching and research institutions in France or abroad, or from public or private research centers.
L'archive ouverte pluridisciplinaire HAL, est destinée au dépôt et à la diffusion de documents scientifiques de niveau recherche, publiés ou non, émanant des établissements d'enseignement et de recherche français ou étrangers, des laboratoires publics ou privés. 


\title{
Non-Markovian transient Casimir-Polder force and population dynamics on excited- and ground-state atoms: Weak- and strong-coupling regimes in generally nonreciprocal environments
}

\author{
George W. Hanson, ${ }^{1, *}$ S. Ali Hassani Gangaraj, ${ }^{2, \dagger}$ Mário G. Silveirinha, ${ }^{3, \ddagger}$ Mauro Antezza, ${ }^{4,5, \S}$ and Francesco Monticone ${ }^{2, \|}$ \\ ${ }^{1}$ Department of Electrical Engineering, University of Wisconsin-Milwaukee, 3200 N. Cramer St., Milwaukee, Wisconsin 53211, USA \\ ${ }^{2}$ School of Electrical and Computer Engineering, Cornell University, Ithaca, New York 14853, USA \\ ${ }^{3}$ Instituto Superior Técnico, University of Lisbon and Instituto de Telecomunicações, Torre Norte, Av. Rovisco Pais 1, \\ Lisbon 1049-001, Portugal \\ ${ }^{4}$ Laboratoire Charles Coulomb (L2C), UMR 5221 CNRS-Université de Montpellier, F-34095 Montpellier, France \\ ${ }^{5}$ Institut Universitaire de France, 1 rue Descartes, F-75231 Paris Cedex 05, France
}

(Received 13 November 2018; published 18 April 2019)

\begin{abstract}
The transient Casimir-Polder force on a two-level atom introduced into a three-dimensional, inhomogeneous, generally nonreciprocal environment is evaluated using non-Markovian Weisskopf-Wigner theory in the strongand weak-coupling regimes. Ground-state and excited atoms are shown to decouple into two separate initialvalue problems, and both the short-time and long-time atomic population and force are evaluated. The results are compared with various Markov approximations of the Weisskopf-Wigner theory and with previous Markov results from the Heisenberg picture.
\end{abstract}

DOI: 10.1103/PhysRevA.99.042508

\section{INTRODUCTION}

The population and vacuum forces on atoms (real or artificial ones) is of fundamental interest and important for practical applications in atomic control [1-3] and quantum information [4]. Particularly for neutral atoms, vacuum forces [5-7] and population-related spontaneous-emission effects play an important role.

In an inhomogeneous environment, spontaneous emission can exert a force on atomic systems. In previous work [8-10], the quantum force and torque on an excited two-level atom in a nonreciprocal environment (a biased plasma interface) was modeled using the Heisenberg picture. It was found that even in a translationally invariant environment, a lateral force can exist due to the nonreciprocal nature of the surface plasmon polaritons (SPPs). The analysis in [8-10] was based on a Markovian solution of the Heisenberg equations of motion (HEM). The Markov approximation (MA), in conjunction with the Sokhotski-Plemelj (SP) identity, allowed the identification of both resonant and nonresonant force contributions $[11,12]$. In the limit $t \rightarrow \infty$, the nonresonant force was shown to be equal to the usual Casimir-Polder (CP) force, which is vertically directed with respect to the interface. The case of short-time dynamics is more delicate, and the previous paper [8] has shown that the MA, together with the use of the SP identity, leads to a nonzero force at the start of the time origin, $t=0$.

\footnotetext{
*george@uwm.edu

†ali.gangaraj@gmail.com

"mario.silveirinha@co.it.pt

§mauro.antezza@umontpellier.fr

"francesco.monticone@ cornell.edu
}

In this work, the correct short-time (transient) behavior of the Casimir-Polder force is determined by removing the Markov approximation, and, in particular, avoiding use of the SP identity. The atom, introduced into an environment at $t=0$, dynamically self-dresses even for a ground-state atom, because its initial state, although an eigenstate of the unperturbed Hamiltonian, is not an eigenstate of the interacting Hamiltonian [13]. For weak coupling, it is found that the fundamental Markov approximation can lead to reasonable results, with the correct force behavior near the time origin, although other commonly used approximations that enable use of the SP identity lead to incorrect short-time behavior.

While there has been a large number of studies on the static CP force (see, e.g., [14,15] and references therein), studies of the transient CP force have been limited in scope (e.g., in [15] a Jaynes-Cummings, single mode field is assumed in the strong-coupling case), and there have been few studies of the non-Markovian CP force [16]. In this work, we consider the initial-value problem of introducing either an excited or a ground-state atom into an environment at $t=0$, which is a special case of the dynamical Casimir effect, which also encompasses photon generation from fast changes in geometry [17]. This problem was considered in [18,19] using an expansion of the Heisenberg equation of motion. Here, we use the Weisskopf-Wigner method, applicable to both weakand strong-coupling regimes, and which rigorously includes non-Markovian effects. We also extend the formulation to nonreciprocal materials (nonreciprocal continuum reservoir), although nonreciprocity is not needed for the studied effects.

We work within the Schrödinger picture, which necessitates elucidating the joint atomic-field states and results in treating the excited-atom and ground-state atom as independent initial-value problems, since the respective states evolve independently. Regarding the Casimir-Polder 
force on a ground-state atom, we show that it arises from non-energy-conserving states. Some parameters are identified to assess the strength of the non-Markovian aspect of the response. The formulation is made for generally nonreciprocal environments, in part to make contact with the work in [8-10], and for applications related to photonic topological insulators, although the main ideas are general and do not necessitate having a nonreciprocal environment.

We now provide a brief comparison of the HEM and Schrödinger picture methods in order to clarify the various approximations used. Both start from the same Hamiltonian. In the HEM, the time evolution of the atomic and field operators is derived as a coupled set of equations from the Heisenberg equation of motion. The solution of the resulting coupled set of equations is extremely difficult, although the field operator equation can be solved by making a one-excitation approximation [20]. However, as this eliminates higher-order correlations, more typically a Markov approximation is made, wherein the dipole operator is assumed to be memoryless. Usually, then, the upper time limit of the spectral integral is approximated as $t \rightarrow \infty$, and the SP identity leads to resonant and nonresonant terms, the latter being a principal-value integral associated with an energy shift of the atomic transition. In [8], we then wrote both contributions in terms of the system Green function, which allows complicated environments (e.g., lossy, inhomogeneous, nonreciprocal) acting as reservoirs to be modeled exactly, in a macroscopic sense. Alternatively, in this work we use the Weisskopf-Wigner method [21-24], which can also incorporate the Green function. In this case, the MA, although also widely used, is not necessary, and the exact solution can be obtained numerically by solving a Volterra integral equation of the second kind. This leads to the non-Markovian (nonexponential) evolution of the population, which can be used in evaluating the exact dipole force. Various MA-type approximations can also be used in approximating the force and are discussed in several Appendixes.

One complication of the Weisskopf-Wigner method is that atom-field product states need to be defined. Considering a two-state atom defining a two-dimensional Hilbert space $H_{a}=\{e, g\}$, and multimode field Fock states $\{0,1,2, \ldots\}$ defining an infinite-dimensional Hilbert space $H_{f}$, where $0,1,2, \ldots$ represents the number of quanta in a generic field mode, the product states $H_{a} \otimes H_{f}$ separate into two groups, $A=\{|e, 0\rangle,|g, 1\rangle,|e, 2\rangle,|g, 3\rangle,|e, 4\rangle, \ldots\}$ and $B=\{|g, 0\rangle,|e, 1\rangle,|g, 2\rangle,|e, 3\rangle,|g, 4\rangle, \ldots\}$ that evolve independently. An initially excited atom evolves within group A and, hence, cannot decay into the ground state of the noninteracting system $|g, 0\rangle$. That is, in the final state the atom can be in the ground state, but the field will have one or more excitations (even in the lossy case). However, the evolution of the noninteracting system ground state can also be determined, where, even starting from the state $|g, 0\rangle$, there is population evolution and force since the directproduct state is not an eigenstate of the full Hamiltonian (except at $t=0$, assuming that the interaction is switched on at that time). Thus, the initially excited-atom case and the ground-state atom case need to be treated as two independent initial-value problems, which is not necessary with the HEM method.
The article is organized as follows. In Sec. II, we describe the generally inhomogeneous, nonreciprocal environment (i.e., a structured reservoir) into which an excited- or ground-state atom is introduced. In Sec. III, we consider introducing an excited atom into the structured reservoir at $t=$ 0 , and we solve for the non-Markovian atomic population in terms of a Volterra integral equation (VIE) of the second kind. We show that the structural form of the VIE is the same as in the reciprocal case, obtained previously, with nonreciprocity simply entering via the Green function. An expression for the non-Markovian force dynamics is then obtained and applied to both weak- and strong-coupling regimes. In particular, transient force dynamics are studied, where it is shown that the force is initially repulsive and then oscillates in sign before settling down to become its static attractive value. For strong coupling to a multimode reservoir, we show Rabi oscillations in the force. In Sec. IV, we repeat the analysis for a groundstate atom, which leads to the transient Casimir-Polder force, exhibiting Rabi oscillations in the strong-coupling regime. Finally, we obtain the long-time dynamics using Laplace transforms and obtain expressions involving a parameter that indicates the degree of non-Markovian behavior. After some some concluding remarks, the Appendixes provide details of the numerical method used to solve the Volterra integral equation and several different Markov-type approximations of the population and force.

\section{NONRECIPROCAL STRUCTURED RESERVOIR ENVIRONMENT}

In a translationally invariant and reciprocal environment, spontaneous emission occurs randomly in all directions so that the net force on a linearly polarized, initially excited atom is zero. For an atom near an interface, the CasimirPolder force is present, associated with vacuum fluctuations and the change of the photonic density of states brought about by the presence of the interface. In addition to the force perpendicular to the interface, as shown in $[8,9]$, at an interface between a nonreciprocal medium and a simple medium, unidirectional surface plasmon polaritons mediate non-null lateral spontaneous-emission forces.

In the following, we consider introducing an excited-state or ground-state atom at $t=0$ into a lossy, inhomogeneous, and nonreciprocal environment, which serves as a structured reservoir for the atom, and examine the time dynamics of the resulting atomic population, spontaneous-emission (SE) rate, and force. The problem is cast as an initial-value problem using Weisskopf-Wigner theory [21-25], adapted for a nonreciprocal medium.

Figure 1 depicts the situation where a two-level atom resides in the vicinity of a material interface.

We suppose the region $z>0$ is filled by vacuum and that the region $z<0$ is filled with a gyrotropic material with permittivity $\boldsymbol{\varepsilon}=\varepsilon_{0}\left(\varepsilon_{t} \mathbf{I}_{t}+\varepsilon_{a} \hat{\mathbf{y}} \hat{\mathbf{y}}+i \varepsilon_{g} \hat{\mathbf{y}} \times \mathbf{I}\right)$, where $\mathbf{I}_{t}=\mathbf{I}-\hat{\mathbf{y}} \hat{\mathbf{y}}$, with $\varepsilon_{g}$ being the magnitude of the gyration pseudovector. For the gyrotropic medium we consider a magnetized plasma (e.g., InSb [26]). For a static bias magnetic field along the $+y$ 


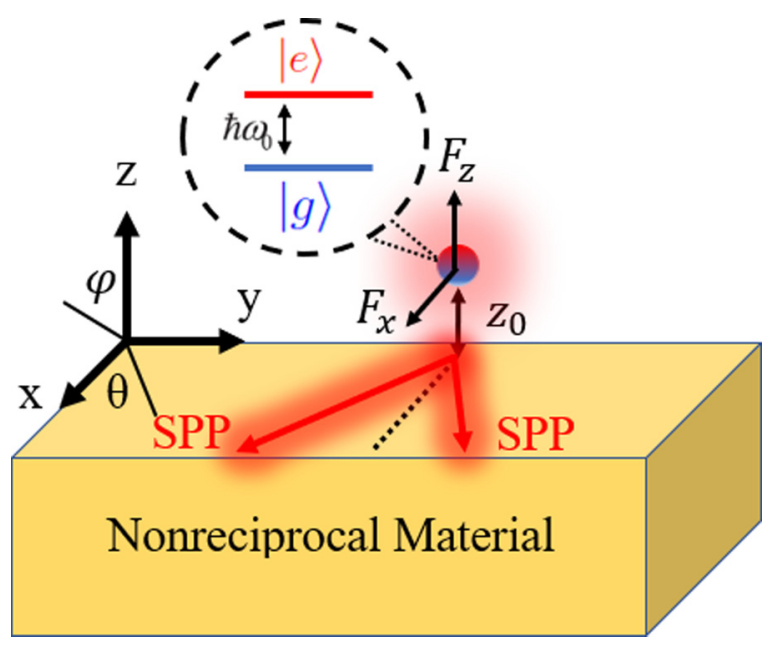

FIG. 1. A two-level system near the surface of a nonreciprocal material, experiencing the Casimir-Polder force, and, for an excited atom, a spontaneous-emission optical force. The main decay channel is the SPPs on the interface.

axis the permittivity components are [27]

$$
\begin{aligned}
\varepsilon_{t} & =1-\frac{\omega_{p}^{2}\left(1+i \Gamma_{c} / \omega\right)}{\left(\omega+i \Gamma_{c}\right)^{2}-\omega_{c}^{2}} \\
\varepsilon_{a} & =1-\frac{\omega_{p}^{2}}{\omega\left(\omega+i \Gamma_{c}\right)}, \quad \varepsilon_{g}=\frac{1}{\omega} \frac{\omega_{c} \omega_{p}^{2}}{\omega_{c}^{2}-\left(\omega+i \Gamma_{c}\right)^{2}} .
\end{aligned}
$$

Here, $\omega_{p}$ is the plasma frequency, $\Gamma_{c}$ is the collision rate associated with damping, $\omega_{c}=-q B_{0} / m>0$ is the cyclotron frequency, $q=-e$ is the electron charge, $m$ is the electron effective mass, and $B_{0}$ is the static bias. In the special case that $B_{0}=0$, the system is reciprocal. A limitingly low-loss plasma is assumed for simplicity, since loss does not qualitatively affect the time dynamics of interest. The analytical form of the Green function for this environment is provided in [8].

In the following, we assume that the dipole is linearly polarized, $\gamma=\hat{\mathbf{z}} \gamma_{z}$, with $\gamma_{z}$ real-valued, located a distance $z_{0}$ from the interface, and we take $\omega_{p}=(2 \pi) 200 \times 10^{12}=$ $1.26 \times 10^{15} / \mathrm{s}$, and $\omega_{0}=0.65 \omega_{p}$.

\section{INITIALLY EXCITED ATOM INTRODUCED INTO A NONRECIPROCAL STRUCTURED RESERVOIR}

In this section, we consider introducing an excited-state atom at $t=0$ into the structured reservoir described above. The ground-state atom is considered in Sec. IV.

\section{A. Initially excited atom: Schrödinger picture wave-function} amplitude evolution in a nonreciprocal environment

In the Schrödinger picture, the system Hamiltonian is [28]

$$
\begin{aligned}
\hat{\mathbf{H}}= & \int d^{3} \mathbf{r} \int_{0}^{\infty} d \omega_{\lambda} \hbar \omega_{\lambda} \hat{\mathbf{f}}^{\dagger}\left(\mathbf{r}, \omega_{\lambda}\right) \hat{\mathbf{f}}\left(\mathbf{r}, \omega_{\lambda}\right) \\
& +\hbar \omega_{0} \hat{\sigma}_{+} \hat{\sigma}_{-}-\hat{\mathbf{p}} \cdot \hat{\mathbf{E}}\left(\mathbf{r}_{0}\right),
\end{aligned}
$$

where the first term is the Hamiltonian for the field modes, the second term is the Hamiltonian for the atomic operators, and the last term accounts for the field-atom coupling. In (2), $\hat{\mathbf{f}}, \hat{\mathbf{f}}^{\dagger}$ are the canonically conjugate field variables (continuum bosonic operator-valued vectors of the combined matter-field system) that satisfy

$$
\begin{array}{r}
{\left[\hat{f}_{k}(\mathbf{r}, \omega), \hat{f}_{k^{\prime}}^{\dagger}\left(\mathbf{r}^{\prime}, \omega^{\prime}\right)\right]=\delta_{k k^{\prime}} \delta\left(\omega-\omega^{\prime}\right) \delta\left(\mathbf{r}-\mathbf{r}^{\prime}\right),} \\
{\left[\hat{f}_{k}(\mathbf{r}, \omega), \hat{f}_{k^{\prime}}\left(\mathbf{r}^{\prime}, \omega^{\prime}\right)\right]=\left[\hat{f}_{k}^{\dagger}(\mathbf{r}, \omega), \hat{f}_{k^{\prime}}^{\dagger}\left(\mathbf{r}^{\prime}, \omega^{\prime}\right)\right]=0,}
\end{array}
$$

where $\hat{\sigma}_{ \pm}$are the canonically conjugate two-level atomic operators $\left(\hat{\sigma}_{+}=|e\rangle\left\langle g\left|, \quad \hat{\sigma}_{-}=\right| g\right\rangle\langle e|=\hat{\sigma}_{+}^{\dagger}\right.$, with $|e\rangle$ and $|g\rangle$ being the excited and ground atomic states, respectively), and $\hat{\mathbf{p}}=\left(\hat{\sigma}_{+}+\hat{\sigma}_{-}\right) \gamma$ is the dipole operator, where $\gamma$ is the dipole operator matrix element.

For the atom-field system, we define product states such as $|e, 0\rangle \equiv|e\rangle \otimes|\{0\}\rangle$ and $\left|g, 1_{i}\left(\mathbf{r}, \omega_{\lambda}\right)\right\rangle \equiv|g\rangle \otimes\left|\left\{1_{i}\left(\mathbf{r}, \omega_{\lambda}\right)\right\}\right\rangle$. The state $\left|1_{i}\left(\mathbf{r}, \omega_{\lambda}\right)\right\rangle=\left|\left\{1_{i}\left(\mathbf{r}, \omega_{\lambda}\right)\right\}\right\rangle$ indicates that the $\lambda$ th field mode of the nonuniform continuum is populated with a single quanta and that it is vector valued with field component in the $i$ th direction. It can be noted that if one uses, rather than the full interaction Hamiltonian $\hat{\mathbf{p}} \cdot \hat{\mathbf{E}}\left(\mathbf{r}_{0}\right)$, the rotating-wave approximation (RWA) interaction Hamiltonian which contains $\left(\hat{\sigma}_{+} \hat{\mathbf{f}}+\right.$ H.c. $)$, then the initial state $|e, 0\rangle$ produces only $|g, 1\rangle$. However, the full interaction Hamiltonian $\hat{\mathbf{p}} \cdot \hat{\mathbf{E}}\left(\mathbf{r}_{0}\right) \sim\left(\hat{\sigma}_{+}+\hat{\sigma}_{-}\right)\left(\hat{\mathbf{f}}+\hat{\mathbf{f}}^{\dagger}\right)$ acting on the initial state $|e, 0\rangle$ leads to an infinite-dimensional Hilbert space of the set of states $A=\{|e, 0\rangle,|g, 1\rangle,|e, 2\rangle,|g, 3\rangle,|e, 4\rangle, \ldots\}$, where the $n>1$ photons could be in the same or different field modes. For the excited atom, we truncate the space to consist of $\{|e, 0\rangle,|g, 1\rangle\}$, which is equivalent to a rotating-wave approximation even when using the full interaction Hamiltonian. Later, we consider non-energy-conserving states, which are necessary for the analysis of the ground-state atom.

We assume a general inhomogeneous, lossy, and nonreciprocal environment characterized by the permittivity tensor $\varepsilon(\mathbf{r}, \omega)$. We follow the phenomenological macroscopic Langevin noise approach [29-33] (see also [34], where a comparison with a generalized Huttner-Barnett approach is discussed, and also [35], where the phenomenological assumptions are derived from a canonical formulation). The quantized Schrödinger picture electric field operator is

$$
\begin{aligned}
\hat{\mathbf{E}}(\mathbf{r})= & \int_{0}^{\infty} d \omega_{\lambda} i \sqrt{\frac{\hbar}{\pi \varepsilon_{0}}} \frac{\omega_{\lambda}^{2}}{c^{2}} \int d^{3} \mathbf{r}^{\prime} \mathbf{G}\left(\mathbf{r}, \mathbf{r}^{\prime}, \omega_{\lambda}\right) \\
& \cdot \mathbf{T}\left(\mathbf{r}^{\prime}, \omega_{\lambda}\right) \cdot \hat{\mathbf{f}}\left(\mathbf{r}^{\prime}, \omega_{\lambda}\right)+\text { H.c. },
\end{aligned}
$$

where $\mathbf{T}\left(\mathbf{r}, \omega_{\lambda}\right) \cdot \mathbf{T}^{\dagger}\left(\mathbf{r}, \omega_{\lambda}\right)=\frac{1}{2 i}\left[\varepsilon(\mathbf{r}, \omega)-\varepsilon^{\dagger}(\mathbf{r}, \omega)\right]$; for reciprocal media, $\mathbf{T}=\sqrt{\operatorname{Im}\{\varepsilon(\mathbf{r}, \omega)\}} \mathbf{I}$, and where $\mathbf{G}\left(\mathbf{r}, \mathbf{r}^{\prime}, \omega_{\lambda}\right)$ is the classical Green function for the nonreciprocal environment, discussed in Appendix A. We assume that an atom is introduced to the environment at $t=0$. Furthermore, we assume zero temperature and that the atomic transition frequency $\omega_{0}$ is not too close to a material resonance. Otherwise, there could be additional transients [36] that are ignored here.

The equation of motion (Schrödinger equation) is $(d / d t)|\psi\rangle=-(i / \hbar) \hat{H}|\psi\rangle$. Using the energy-conserving states (ECS) $\left\{|e, 0\rangle,\left|g, 1_{i}\left(\mathbf{r}, \omega_{\lambda}\right)\right\rangle\right\}$, the expansion of the wave 
function is

$$
\begin{aligned}
|\psi(t)\rangle_{\mathrm{ECS}} & =b_{e o}(t)|e, 0\rangle \\
& +\int d^{3} \mathbf{r} \int_{0}^{\infty} d \omega_{\lambda} b_{g 1 i}\left(\mathbf{r}, \omega_{\lambda}, t\right)\left|g, 1_{i}\left(\mathbf{r}, \omega_{\lambda}\right)\right\rangle,
\end{aligned}
$$

where $b_{e o}(t)$ is the atomic excited-state population amplitude. Here and in the following we sum over repeated vectorcomponent indices. Conservation of probability requires

$$
\left|b_{e o}(t)\right|^{2}+\int_{0}^{\infty} d \omega_{\lambda} \int d \mathbf{r}\left|b_{g i}\left(\mathbf{r}, \omega_{\lambda}, t\right)\right|^{2}=1 .
$$

It is convenient to write $b_{e o}(t)=c_{e o}(t) e^{-i \omega_{0} t}$ and $b_{g 1 i}\left(\mathbf{r}, \omega_{\lambda}, t\right)=c_{g 1 i}\left(\mathbf{r}, \omega_{\lambda}, t\right) e^{-i \omega_{\lambda} t}$. Plugging the wave function into the Schrödinger equation and using orthogonality, for $\gamma=\hat{\mathbf{x}}_{j} \gamma_{j}$, it is straightforward to obtain the coupled set of equations ( $j$ is fixed):

$$
\begin{aligned}
\frac{d}{d t} c_{e o}(t)= & -\gamma_{j} \sqrt{\frac{1}{\hbar \pi \varepsilon_{0}}} \int_{0}^{\infty} d \omega_{\lambda} \frac{\omega_{\lambda}^{2}}{c^{2}} \\
& \times \int d^{3} \mathbf{r}^{\prime} K_{j i}\left(\mathbf{r}_{0}, \mathbf{r}^{\prime}, \omega_{\lambda}\right) c_{g 1 i}\left(\mathbf{r}^{\prime}, \omega_{\lambda}, t\right) e^{-i\left(\omega_{\lambda}-\omega_{0}\right) t},
\end{aligned}
$$

$$
\begin{aligned}
\frac{d}{d t} c_{g 1 i}\left(\mathbf{r}, \omega_{\lambda}, t\right)= & \sqrt{\frac{1}{\hbar \pi \varepsilon_{0}}} \gamma_{j} \frac{\omega_{\lambda}^{2}}{c^{2}} K_{j i}^{*}\left(\mathbf{r}_{0}, \mathbf{r}, \omega_{\lambda}\right) \\
& \times c_{e o}(t) e^{i\left(\omega_{\lambda}-\omega_{0}\right) t},
\end{aligned}
$$

where $\mathbf{K}\left(\mathbf{r}, \mathbf{r}^{\prime}, \omega_{\lambda}\right)=\mathbf{G}\left(\mathbf{r}, \mathbf{r}^{\prime}, \omega_{\lambda}\right) \cdot \mathbf{T}\left(\mathbf{r}^{\prime}, \omega_{\lambda}\right)$. It can be noted that (8)-(9) are the same as [[28], (6.26)-(6.27)] and [[31], (23)-(24)], except here generalized to nonreciprocal media.

Integrating (9), assuming that the excitation initially resides in the atom, $c_{g 1 i}\left(\mathbf{r}, \omega_{\lambda}, t=0\right)=0$, and inserting the result into (8) and using (A3) leads to the non-Markovian population equation in the form of a Volterra integral equation of the second kind,

$$
\frac{d}{d t} c_{e o}(t)=\int_{0}^{t} H\left(t, t^{\prime}\right) c_{e o}\left(t^{\prime}\right) d t^{\prime},
$$

with the kernel

$$
\begin{aligned}
H\left(t, t^{\prime}\right)= & -\frac{1}{\hbar \pi \varepsilon_{0}} \int_{0}^{\infty} d \omega_{\lambda} \frac{\omega_{\lambda}^{2}}{c^{2}} \frac{\gamma \cdot \mathbf{G}_{\mathbf{I}}\left(\mathbf{r}_{0}, \mathbf{r}_{0}, \omega_{\lambda}\right) \cdot \gamma}{2 i} \\
& \times e^{-i\left(\omega_{\lambda}-\omega_{0}\right)\left(t-t^{\prime}\right)},
\end{aligned}
$$

where $G_{\mathbf{I}, i, j}\left(\mathbf{r}, \mathbf{r}_{0}, \omega_{\lambda}\right)=G_{i j}\left(\mathbf{r}, \mathbf{r}_{0}, \omega_{\lambda}\right)-G_{i j}^{*}\left(\mathbf{r}_{0}, \mathbf{r}, \omega_{\lambda}\right)[32]$. We will assume the initial-value condition $c_{e o}(0)=1$. It is useful to note that for a linearly polarized, real-valued dipole moment (assumed here), $\gamma \cdot \mathbf{G}_{\mathbf{I}}\left(\mathbf{r}_{0}, \mathbf{r}_{0}, \omega_{\lambda}\right) \cdot \gamma$ picks out a diagonal element of the Green function, and $G_{\mathbf{I}, i i}\left(\mathbf{r}_{0}, \mathbf{r}_{0}, \omega\right)=$ $2 i \operatorname{Im} G_{i i}\left(\mathbf{r}_{0}, \mathbf{r}_{0}, \omega\right)$, even for a nonreciprocal medium; so in that case the form of the Volterra equation (10) is the same in the reciprocal and nonreciprocal cases. The procedure for numerically solving the Volterra integral equation is shown in Appendix B. Appendix C details various levels of Markov approximations that enable closed-form solutions. Specifically, if the population is assumed to be memoryless [Markov approximation (MA)], $c_{e o}\left(t^{\prime}\right) \simeq c_{e o}(t)$, the upper limit of the

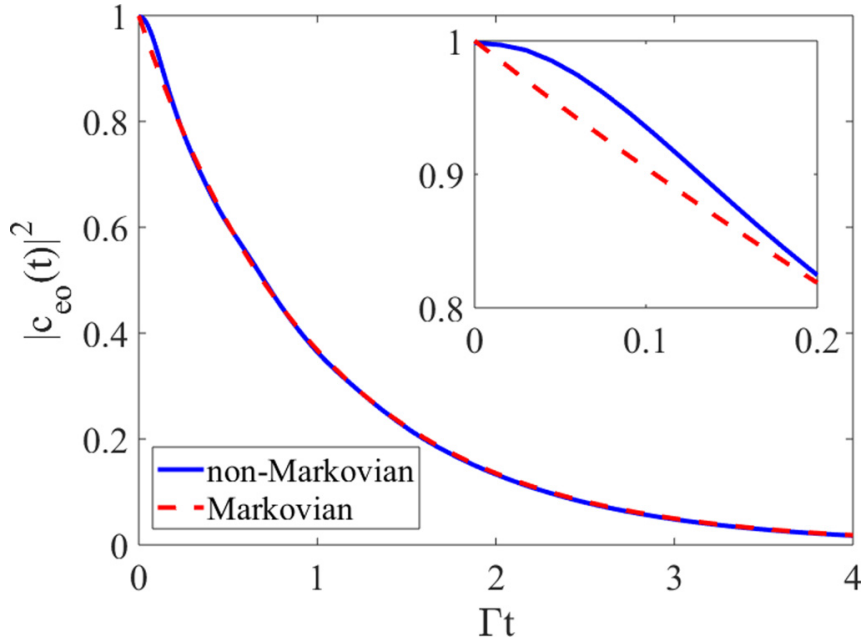

FIG. 2. Non-Markovian population dynamics in the weakcoupling regime, obtained from the numerical solution of (10) and compared with the usual Markov population decay using (C6). The insert shows the behavior near $t=0$. The atom is located $z_{0}=$ $0.7 c / \omega_{p}$ above the interface, such that $g=0.0455$.

time integral is extended to infinity and the Sokhotski-Plemelj (SP) identity (C1) is used. We call this the full Markov (FM) approximation. If, however, the MA is made but the upper limit of the integration is not extended to infinity, we call this the partial Markov (PM) approximation.

In order to quantify the degree of coupling, we introduce the coupling parameter

$$
g=\frac{|\gamma|}{\hbar \omega_{\mathrm{SPP}}} \sqrt{\frac{\hbar \omega_{\mathrm{SPP}}}{32 \pi \varepsilon_{0} z_{0}^{3}}},
$$

which was obtained for the unbiased, quasistatic case and which can be used to give a order-of-magnitude quantification of the coupling strength for the biased case. In (12) we assume $\omega_{\mathrm{SPP}}=\omega_{p} / \sqrt{2}$, which corresponds to the unbiased case. The coupling parameter delineates weak $(g \ll 1)$ and strong $(g \geqslant$ $0.5)$ coupling.

Figure 2 shows the non-Markovian population dynamics obtained from the numerical solution of (10) for a dipole positioned $z_{0}=0.7 c / \omega_{p}$ above the interface, such that $g=$ 0.0455 indicates weak coupling and $\Gamma=1.53 \times 10^{13} / \mathrm{s}$ from (C5). Comparison is made to the FM approximation (C6) [the PM approximation, Eq. (C8), yields similar results]. The non-Markovian result shows the correct zero slope at $t=0$ [37-39], as shown in the insert of Fig. 2. Other than the initial slope, it can be seen that excellent agreement between the Markov approximation and the non-Markov solution is obtained, as expected for weak coupling. Although not shown, the non-Markovian solution is also expected to show slower than exponential decay for long times [40].

Figure 3 shows the same result as Fig. 2, except for atom height $z_{0}=0.1 c / \omega_{p}$ above the interface. In this case, $g=$ 0.8427 , indicating strong coupling, and $\Gamma=3.64 \times 10^{15} / \mathrm{s}$ from (C5). The exact solution is strongly non-Markovian, as expected, and exhibits Rabi oscillations. 


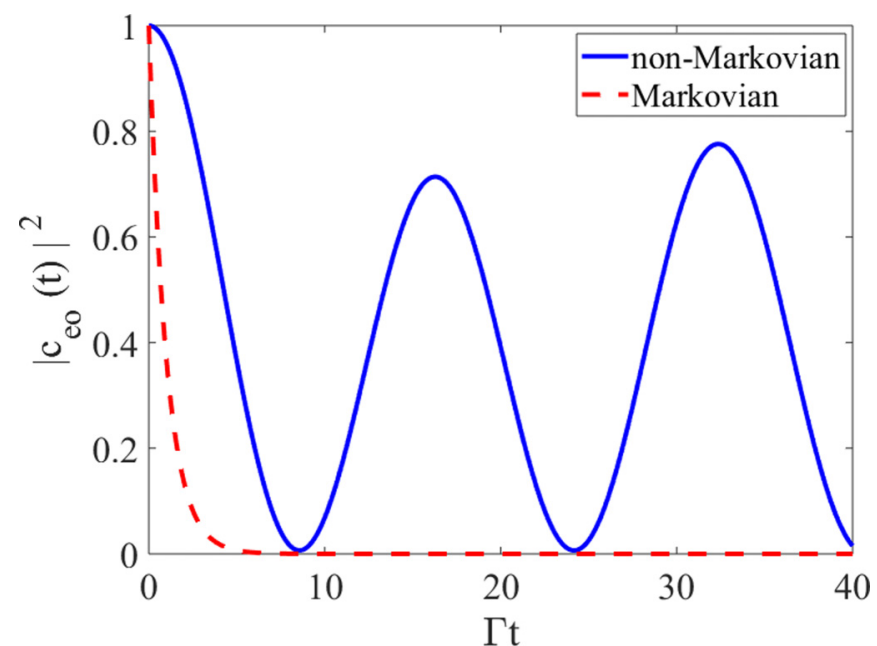

FIG. 3. Non-Markovian population dynamics in the strongcoupling regime, obtained from the numerical solution of (10) and compared with the usual Markov population decay using (C6). The atom is located $z_{0}=0.1 c / \omega_{p}$ above the interface, such that $g=$ 0.8427 .

\section{B. Initially excited atom: Transient non-Markovian \\ Casimir-Polder force in a nonreciprocal environment}

From canonical quantization, the quantum operator for the dipole force on an atom located at $\mathbf{r}_{0}$ is [41]

$$
\hat{\mathscr{F}}_{j}=\left.\hat{\mathbf{p}} \cdot \frac{\partial}{\partial j} \hat{\mathbf{E}}(\mathbf{r})\right|_{\mathbf{r}=\mathbf{r}_{0}}, j=x, y, z .
$$

The expectation value of force operator in the $\alpha$ th direction due to a dipole oriented along the $j$ th coordinate is

$$
\begin{aligned}
\mathscr{F}_{\alpha}^{j}(t) & =\left\langle\hat{\mathscr{F}}_{\alpha}{ }^{j}\right\rangle \\
& =\left\langle\psi(t)\left|\left(\left.\left(\hat{\sigma}_{+}+\hat{\sigma}_{-}\right) \gamma_{j} \hat{\mathbf{x}}_{j} \cdot \partial_{\alpha} \hat{\mathbf{E}}(\mathbf{r})\right|_{\mathbf{r}=\mathbf{r}_{0}}\right)\right| \psi(t)\right\rangle .
\end{aligned}
$$

Using (9), (A3), and summing over repeated indices, the general non-Markovian force is

$$
\begin{aligned}
\mathscr{F}_{\alpha}(t) & 2 \operatorname{Re}\left\{\left.\frac{i}{\pi \varepsilon_{0}} c_{e o}^{*}(t) \int_{0}^{\infty} d \omega_{\lambda} \frac{\omega_{\lambda}^{2}}{c^{2}} \frac{\partial}{\partial \alpha} \frac{\gamma \cdot \mathbf{G}_{\mathbf{I}}\left(\mathbf{r}, \mathbf{r}_{0}, \omega_{\lambda}\right) \cdot \gamma}{2 i}\right|_{\mathbf{r}=\mathbf{r}_{0}}\right. \\
\times & \left.\int_{0}^{t} c_{e o}\left(t^{\prime}\right) e^{-i\left(\omega_{\lambda}-\omega_{0}\right)\left(t-t^{\prime}\right)} d t^{\prime}\right\}
\end{aligned}
$$

for $\alpha=x, y, z$. This is the first main analytical result of this paper.

Various Markov approximations of the force are provided in Appendix D. In particular, one can substitute the FM or PM approximations for the population into the force expression and then evaluate the resulting time integral exactly, leading to what we refer to as the FM or PM approximation, respectively, of the force. Alternatively, one could impose the Markov approximation $c_{e o}\left(t^{\prime}\right) \simeq c_{e o}(t)$ directly in the time integral in the force expression and then either evaluate the resulting time integral exactly, which we denote as the PM2 approximation, or extend the upper limit of the time integral to infinity and use the Sokhotski-Plemelj identity, which we denote as the FM2 approximation.

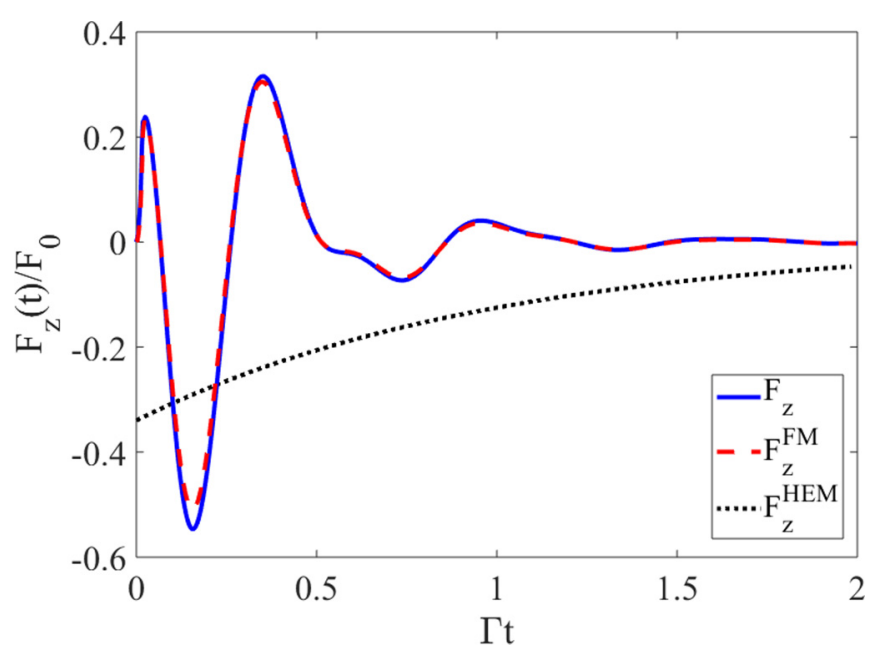

FIG. 4. Normalized non-Markovian vertical force (15) in the weak-coupling regime compared with the Markov approximation (D1) and the HEM result [8]. $\mathscr{F}_{0}=3|\gamma|^{2} /\left(16 \pi z_{0}^{4} \varepsilon_{0}\right)(\mathrm{N})$. The atom is located $z_{0}=0.7 c / \omega_{p}$ above the interface, $g=0.0455$.

Figure 4 shows the normalized exact vertical force $\mathscr{F}_{z}$ from (15) compared with the FM approximation (D1) and the result from [8]. Note that the force is initially repulsive and then oscillates in sign before settling down to become attractive.

The FM approximation is in good agreement with the exact force (15), indicating that the short-time force dynamics are essentially Markovian in the weak-coupling case. Importantly, this approximation does not entail use of the SP identity and has the correct null value at the time origin [42]. All solutions initially oscillate and then eventually settle down to the MA HEM solution, which was obtained in [8] using the SP identity (which does not provide the correct short-time dynamics). For the nonreciprocal case, a lateral force also exists but will be omitted here.

Figure 5 shows the normalized exact vertical force $\mathscr{F}_{z}$ from (15) compared with the Markovian HEM result [8] for the strong-coupling case, $z_{0}=0.1 c / \omega_{p}$. The Rabi oscillations of the population (Fig. 3) are evident in the force, indicating strongly non-Markovian behavior.

\section{CASIMIR-POLDER FORCE ON A GROUND-STATE ATOM INTRODUCED INTO A NONRECIPROCAL STRUCTURED RESERVOIR}

In the Heisenberg picture, atom-field states do not need to be defined, and the force $\mathscr{F}_{z}$ found via the HEM naturally becomes the Casimir-Polder force for large times. However, using the Weisskopf-Wigner method, the states for the excitedatom field are $\left\{|e, 0\rangle,\left|g, 1_{i}\left(\mathbf{r}, \omega_{\lambda}\right)\right\rangle\right\}$, and the joint atom-field ground state is never reached (even using the full set of states $A=\{|e, 0\rangle,|g, 1\rangle,|e, 2\rangle,|g, 3\rangle,|e, 4\rangle, \ldots\})$. In this section, we investigate the $\mathrm{CP}$ force on a ground-state atom introduced into a nonreciprocal structured reservoir at $t=0$. We will continue to assume a vertically polarized atom, although for the ground state a better approximation would be to average over vertical and horizontal polarizations. 


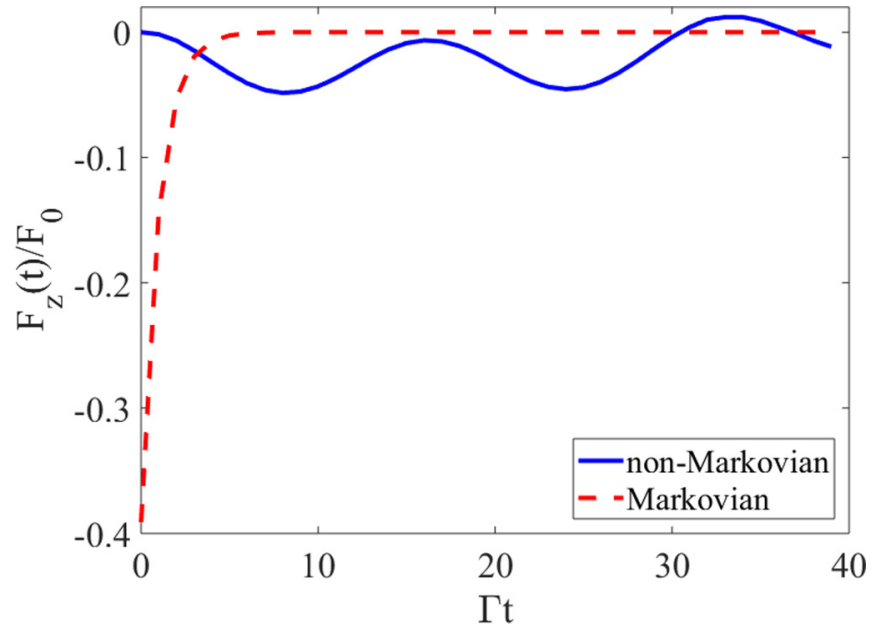

FIG. 5. Normalized non-Markovian vertical force (15) in the strong-coupling regime compared with the Markovian HEM result [8]. $\mathscr{F}_{0}=3|\gamma|^{2} /\left(16 \pi z_{0}^{4} \varepsilon_{0}\right)(\mathrm{N})$. The atom is located $z_{0}=0.1 c / \omega_{p}$ above the interface, $g=0.8427$.

\section{A. Ground-state atom: Non-Markovian population and transient Casimir-Polder force in a nonreciprocal environment}

When considering the Casimir-Polder force on a groundstate atom, the assumption is usually that both the atom and field are in the ground state. If we assume an initial state as a direct product of atomic and field ground states, i.e., the noninteracting system ground state $|g, 0\rangle$, the full interaction Hamiltonian acts on the initial state to produce the set of states $\{|g, 0\rangle,|e, 1\rangle,|g, 2\rangle,|e, 3\rangle,|g, 4\rangle, \ldots\}$, where, again, the numbers represent the number of quanta in the generic field mode. The two sets of states $A=$ $\{|e, 0\rangle,|g, 1\rangle,|e, 2\rangle,|g, 3\rangle,|e, 4\rangle, \ldots\}$, used for an initially excited atom, and $B=\{|g, 0\rangle,|e, 1\rangle,|g, 2\rangle,|e, 3\rangle,|g, 4\rangle, \ldots\}$, used for an initial ground-state atom, are independent (uncoupled). The set $B$ is useful for the following situation: If we introduce a quasi-ground-state atom $|g, 0\rangle$ at $t=0$ into a structured nonreciprocal reservoir, then the SE and force evolve using set $B$, in contradistinction to the situation involving an initially excited atom considered in the previous sections. Here we truncate the Hilbert space to consist of the two non-energy-conserving (NEC) virtual states $\left\{|g, 0\rangle,\left|e, 1_{i}\left(\mathbf{r}, \omega_{\lambda}\right)\right\rangle\right\}$, such that the wave function is

$$
\begin{aligned}
|\psi(t)\rangle_{\mathrm{NECS}}= & b_{g o}(t)|g, 0\rangle \\
& +\int d^{3} \mathbf{r} \int_{0}^{\infty} d \omega_{\lambda} b_{e 1 i}\left(\mathbf{r}, \omega_{\lambda}, t\right)\left|e, 1_{i}\left(\mathbf{r}, \omega_{\lambda}\right)\right\rangle .
\end{aligned}
$$

Since the two pairs of states $A$ and $B$ are independent (uncoupled), $|\psi(t)\rangle_{\mathrm{ECS}}$ and $|\psi(t)\rangle_{\mathrm{NECS}}$ can be evolved separately.

For the NECS states $\left\{|g, 0\rangle,\left|e, 1_{i}\left(\mathbf{r}, \omega_{\lambda}\right)\right\rangle\right\}$ we find that the population satisfies the second-kind Volterra integral equation

where

$$
\frac{d}{d t} b_{g o}(t)=\int_{0}^{t} H\left(t, t^{\prime}\right) b_{g o}\left(t^{\prime}\right) d t^{\prime},
$$

$$
\begin{aligned}
H\left(t, t^{\prime}\right)= & -\frac{1}{\hbar \pi \varepsilon_{0}} \int_{0}^{\infty} d \omega_{\lambda} \frac{\omega_{\lambda}^{2}}{c^{2}} \frac{\gamma \cdot \mathbf{G}_{\mathbf{I}}\left(\mathbf{r}_{0}, \mathbf{r}_{0}, \omega_{\lambda}\right) \cdot \gamma}{2 i} \\
& \times e^{-i\left(\omega_{\lambda}+\omega_{0}\right)\left(t-t^{\prime}\right)},
\end{aligned}
$$

assuming $b_{e 1 i}\left(\mathbf{r}, \omega_{\lambda}, t=0\right)=0$, and the initial-value condition $b_{g o}(0)=1$. Comparing the kernels, Eqs. (11) and (18), we see that they are the same except that $\left(\omega_{\lambda}-\omega_{0}\right)$ in (11) is replaced by $\left(\omega_{\lambda}+\omega_{0}\right)$ in (18). Whereas the Markov approximation of Eqs. (10) and (11) leads to both exponential decay and an energy shift (Appendix D), the Markov approximation of Eqs. (17) and (18) leads to only an energy shift.

Similar to (15), the non-Markovian force on the groundstate atom is

$$
\mathscr{F}_{\alpha}(t)=2 \operatorname{Re}\left\{\left.\frac{i}{\pi \varepsilon_{0}} b_{g o}^{*}(t) \int_{0}^{\infty} d \omega_{\lambda} \frac{\omega_{\lambda}^{2}}{c^{2}} \frac{\partial}{\partial \alpha} \frac{\gamma \cdot \mathbf{G}_{\mathbf{I}}\left(\mathbf{r}, \mathbf{r}_{0}, \omega_{\lambda}\right) \cdot \gamma}{2 i}\right|_{\mathbf{r}=\mathbf{r}_{0}} \int_{0}^{t} b_{g o}\left(t^{\prime}\right) e^{-i\left(\omega_{\lambda}+\omega_{0}\right)\left(t-t^{\prime}\right)} d t^{\prime}\right\}
$$

for $\alpha=x, y, z$. Together with (15), this is one of the main results of this paper.

The non-Markovian population of the ground-state atom is obtained by the numerical solution of (17) using the procedure described in Appendix B [although, due to the rapidly oscillating temporal integral in (17), a much smaller time step needs to be used compared to solving (10)]. Various Markov approximations of the population and force are provided in Appendixes $\mathrm{C}$ and $\mathrm{D}$, respectively.

For the weak-coupling case, $b_{g o}(t) \simeq e^{i \delta_{g} t}$, and so $\left|b_{g o}(t)\right|^{2} \simeq 1$. The frequency shift (Appendix $\mathrm{C}$ ) is found to be $\delta_{g}=7.78 \times 10^{-4} \omega_{0}$, such that the real and imaginary parts of the population oscillate with a period of $\Gamma \mathrm{T} \simeq 151.55$, where, for reference, $\Gamma$ is the decay rate of the excited atom, (C5). Alternatively, in the strong-coupling case, there are Rabi oscillations as well as a frequency shift, $\delta_{g}=0.32 \omega_{0}$, which leads to a period of $\Gamma \mathrm{T} \simeq 0.4$. Figure 6 shows $\left|b_{g o}(t)\right|^{2}$, where it can be seen that the population is strongly non-Markovian, and exhibits Rabi oscillations.

The exact, generally non-Markovian force is obtained by using the numerically determined amplitude $b_{g o}(t)$ from (17) in (19). The vertical force (19) is shown in Fig. 7 for the weak-coupling case, along with the Markov approximation (D4) [(D5) is essentially the same as (D4)] and compared with the FM2 approximation (D6). We see that at $t=0$ the force has the correct null value and then oscillates and rapidly settles down to the value of the FM2 approximation, which is the usual static CP force. The FM2 approximation does not have the correct value at $t=0$ due to extending upper limit of the time integral to $t \rightarrow \infty$. Therefore we see that the longtime $(\Gamma t \gg 1)$ behavior of the vertical force on the groundstate atom is the usual Markovian Casimir-Polder force. 


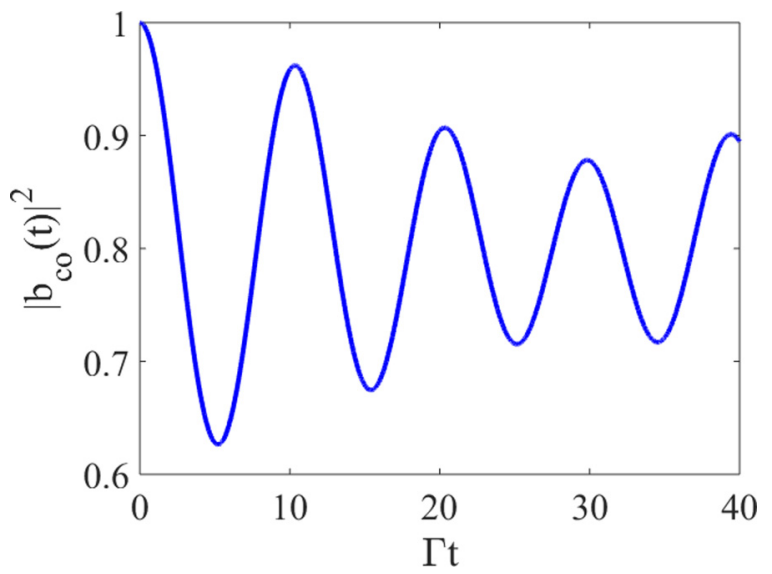

FIG. 6. Non-Markovian ground-state atom population dynamics in the strong-coupling regime, obtained from the numerical solution of (17). The atom is located $z_{0}=0.1 c / \omega_{p}$ above the interface such that $g=0.8427$.

The reasons for the oscillations in Fig. 7 are as follows. Since we take a bare-state rather than dressed-state approach, the initial state $|g, 0\rangle$ is not the true ground state of the atom (and, certainly, neither is $|e, 1\rangle$ ). As such, the (light-matter) interaction can push the atom to other states, but with time the system finally settles down into a final state that locally approximates the true ground state.

The vertical force in the strong-coupling regime is shown in Fig. 8. The strong oscillations in the force are due to the Rabi oscillations of the population.

Non-Markovian Casimir-Polder force for $t \rightarrow \infty$ on a ground-state atom

In the previous section, the non-Markovian population and $\mathrm{CP}$ force on a ground-state atom in a nonreciprocal structured reservoir was determined numerically (and a Markov approximation is provided in Appendix C). Next, we consider the exact $t \rightarrow \infty$ behavior of the population and force on a

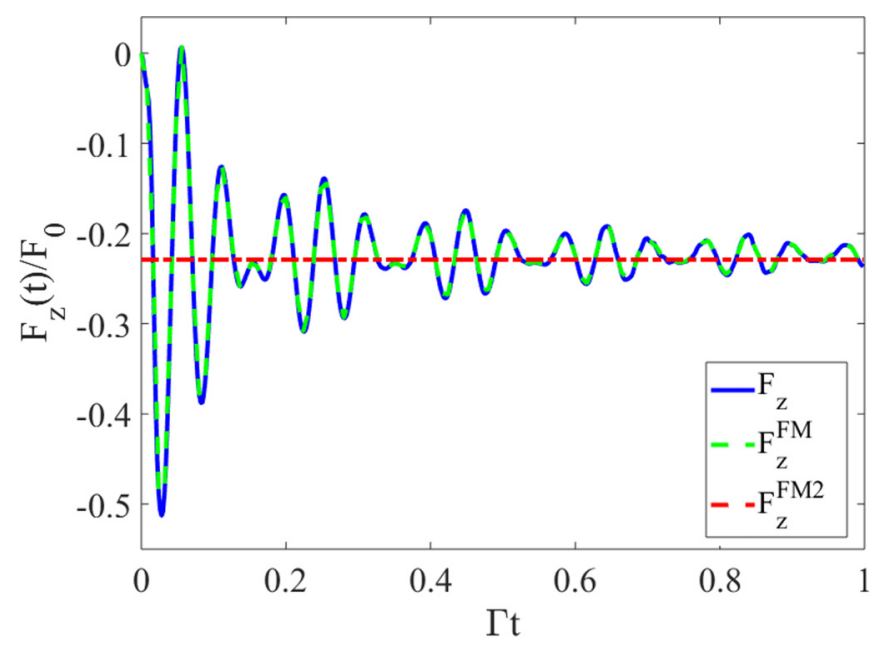

FIG. 7. Vertical force dynamics (transient Casimir-Polder force) on a ground-state atom in the weak-coupling regime. The atom is located $z_{0}=0.7 c / \omega_{p}$ above the interface such that $g=0.0455$.

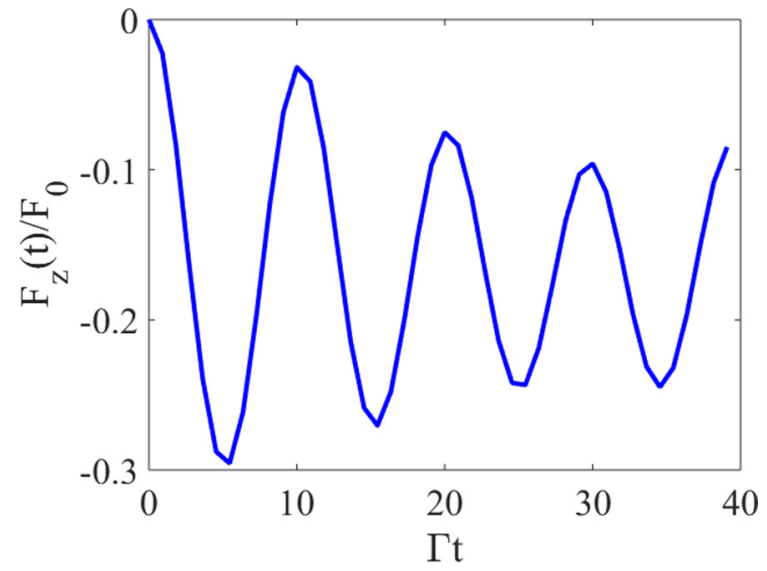

FIG. 8. Vertical force dynamics (transient Casimir-Polder force) on a ground-state atom in the strong-coupling regime $\left(z_{0}=0.1 c / \omega_{p}\right.$, $g=0.8427)$.

ground-state (direct-product ground state) atom. This leads to a method to quantify the level of the non-Markovian behavior.

Starting with the energy-nonconserving states associated with $|g, 0\rangle$, the non-Markovian population obeys ((17)) and (18), which have a convolution form. Taking Laplace transforms,

$$
\begin{aligned}
& s b_{g o}(s)-b_{g o}\left(t=0^{+}\right) \\
& =-\frac{1}{\hbar \pi \varepsilon_{0}} \int_{0}^{\infty} d \omega_{\lambda} \frac{\omega_{\lambda}^{2}}{c^{2}} \frac{\gamma \cdot \mathbf{G}_{\mathbf{I}}\left(\mathbf{r}_{0}, \mathbf{r}_{0}, \omega_{\lambda}\right) \cdot \gamma}{2 i} b_{g o}(s) \mathscr{L},
\end{aligned}
$$

where

$$
\begin{aligned}
\mathscr{L}=\mathscr{L}\left\{e^{-i\left(\omega_{\lambda}+\omega_{0}\right) t}\right\} & =\int_{0}^{\infty} e^{-i\left(\omega_{\lambda}+\omega_{0}\right) t} e^{-s t} d t \\
& =\frac{1}{s+i\left(\omega_{\lambda}+\omega_{0}\right)} .
\end{aligned}
$$

Therefore,

$$
b_{g o}(s)=\frac{b_{g o}\left(t=0^{+}\right)}{s+\Gamma(s)}=\frac{1}{s+\Gamma(s)},
$$

where

$$
\Gamma(s)=\frac{1}{\hbar \pi \varepsilon_{0}} \int_{0}^{\infty} d \omega_{\lambda} \frac{\omega_{\lambda}^{2}}{c^{2}} \frac{\gamma \cdot \mathbf{G}_{\mathbf{I}}\left(\mathbf{r}_{0}, \mathbf{r}_{0}, \omega_{\lambda}\right) \cdot \gamma}{2 i} \mathscr{L} .
$$

Replacing $s \rightarrow s^{\prime}-i \omega_{0}$,

$$
b_{g o}(t)=\frac{e^{-i \omega_{0} t}}{2 \pi i} \int_{\delta-i \infty}^{\delta+i \infty} \frac{1}{s^{\prime}-i \omega_{0}+G\left(s^{\prime}\right)} e^{s^{\prime} t} d s^{\prime},
$$

where $G\left(s^{\prime}\right)=\Gamma\left(s^{\prime}-i \omega_{0}\right)$. It can be seen that $G\left(s^{\prime}\right)$ has logarithmic-type branch points at $s^{\prime}=0$ and $s^{\prime}=-i \infty$. To see that a branch cut (BC) exists from $s^{\prime}=0$ to $s^{\prime}=-i \infty$, we can consider $[38,39] G_{d}\left(s^{\prime}\right)=G(x+i y)-G(-x+i y)$ :

$$
\begin{aligned}
& \lim _{x \rightarrow 0} G_{d}\left(s^{\prime}\right) \\
& \quad=\frac{2}{\hbar \varepsilon_{0}} \int_{0}^{\infty} d \omega_{\lambda} \frac{\omega_{\lambda}^{2}}{c^{2}} \frac{\gamma \cdot \mathbf{G}_{\mathbf{I}}\left(\mathbf{r}_{0}, \mathbf{r}_{0}, \omega_{\lambda}\right) \cdot \gamma}{2 i} \delta\left(y+\omega_{\lambda}\right)
\end{aligned}
$$




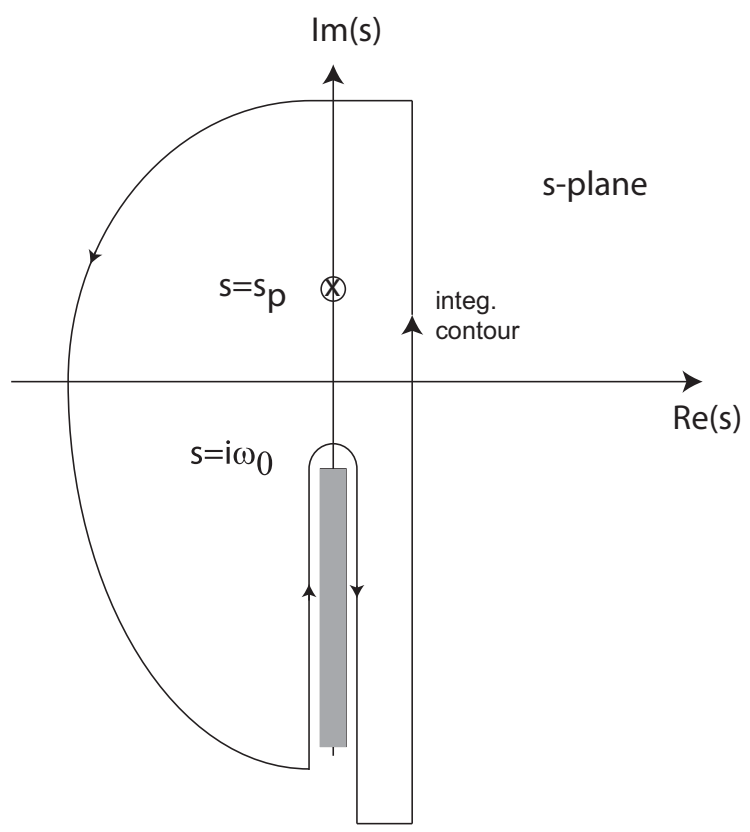

FIG. 9. Depiction of the $s$ plane showing the pole, branch cut, and integration contour.

using

$$
\delta(y)=\frac{1}{\pi} \lim _{x \rightarrow 0} \frac{x}{y^{2}+x^{2}} .
$$

For $y>0$, the $\delta$ function is never encountered, and so $G_{d}(y)=$ 0 , and there is no discontinuity. But, for $y<0$, the $\delta$ function is encountered and so the branch cut goes from $s^{\prime}=0$ to $s^{\prime}=-i \infty$. Since $s=s^{\prime}-i \omega_{0}$, the BC goes from $s=-i \omega_{0}$ to $s=-i \infty$.

Poles will occur at $s+\Gamma(s)=0$. For the numerical parameters assumed in Sec. II, it is found that there is one pole located on the imaginary axis at $s_{p}=i \alpha_{p}$, and $\alpha_{p} / \omega_{0} \ll 1$.

The complex $s$ plane is depicted in Fig. 9, showing that the inverse Laplace transform will involve a residue and a branchcut integral:

$$
\begin{aligned}
b_{g o}(t) & =\frac{1}{2 \pi i} \int_{\delta-i \infty}^{\delta+i \infty} b_{g o}(s) e^{s t} d s \\
& =b_{g o}^{\mathrm{Res}}(t)+\frac{1}{2 \pi i} \int_{\mathrm{BC}} \frac{1}{s+\Gamma(s)} e^{s t} d s
\end{aligned}
$$

where

$$
b_{g o}^{\operatorname{Res}}(t)=\frac{2 \pi i}{2 \pi i} \frac{1}{\Gamma^{\prime}\left(s_{p}\right)} e^{s_{p} t}=c e^{i \alpha_{p} t} .
$$

By the Riemann-Lebesgue lemma, the branch-cut contribution goes to zero as $t \rightarrow \infty$ so that $b_{g o}(t \rightarrow \infty)=b_{g o}^{\text {Res }}(t)$. This can be compared to (C12), with the difference being the value of the oscillation frequency $\delta_{g}$ in $(\mathrm{C} 12)$ and $\alpha_{p}$ in (29).

Having considered the population, we want to evaluate the $t \rightarrow \infty$ value of the force (19). The method of directly evaluating this using Laplace transforms is cumbersome, and so we will, instead, insert the population obtained above, $\lim _{t \rightarrow \infty} b_{g o}(t)=b_{g o}^{\mathrm{Res}}(t)=c e^{i \alpha_{p} t}$, into (19), leading to

$$
\begin{aligned}
& \mathscr{F} \mathscr{C}_{\alpha} \\
& =|c|^{2} 2 \operatorname{Re}\left\{\left.\frac{i}{\pi \varepsilon_{0}} \int_{0}^{\infty} d \omega_{\lambda} \frac{\omega_{\lambda}^{2}}{c^{2}} \frac{\partial}{\partial \alpha} \frac{\gamma \cdot \mathbf{G}_{\mathbf{I}}\left(\mathbf{r}, \mathbf{r}_{0}, \omega_{\lambda}\right) \cdot \gamma}{2 i}\right|_{\mathbf{r}=\mathbf{r}_{0}}\right. \\
& \left.\quad \times \frac{1}{\omega_{\lambda}+\omega_{0}+\alpha_{p}}\right\} .
\end{aligned}
$$

Therefore, there is only a nonresonant component of the exact non-Markovian Casimir-Polder force on the ground-state atom.

Comparing with the FM approximation obtained by the same method, (D4), in the $t \rightarrow \infty$ limit,

$$
\begin{aligned}
\mathscr{F}_{\alpha}^{\mathrm{FM}}= & 2 \operatorname{Re}\left\{\frac{i}{\pi \varepsilon_{0}} \int_{0}^{\infty} d \omega_{\lambda} \frac{\omega_{\lambda}^{2}}{c^{2}} \frac{\partial}{\partial \alpha} \frac{\gamma \cdot \mathbf{G}_{\mathbf{I}}\left(\mathbf{r}, \mathbf{r}_{0}, \omega_{\lambda}\right) \cdot \gamma}{2 i}\right. \\
& \left.\times \frac{1}{\omega_{\lambda}+\omega_{0}+\delta_{g}}\right\}
\end{aligned}
$$

we see that if $|c|=1$ and $\alpha_{p}=\delta_{g}$ (the Lamb shift), then these are the same. The occurrence of $\alpha_{p} \neq \delta_{g}$ and $|c| \neq 1$ differentiates the Markov and non-Markov solutions.

Numerically, for weak coupling $\left(z_{0}=0.7 c / \omega_{p}\right)$ at $\omega_{0}=0.65 \omega_{p}, \quad \alpha_{p}=7.80 \times 10^{-4} \omega_{0}, \quad$ which agrees with the frequency-shift FM approximation, $\delta_{g}=7.78 \times 10^{-4} \omega_{0}$. Furthermore,

$$
|c|=\left|b_{g o}^{\operatorname{Res}}(t)\right|=\frac{1}{\left|D^{\prime}\left(s_{p}\right)\right|}=0.9995,
$$

so we have, for the pole, $\alpha_{p} \simeq \delta_{g} \ll \omega_{0}$ and $|c| \simeq 1$, in which case the non-Markovian $t \rightarrow \infty$ result (30) is approximately the same as the FM result for $t \rightarrow \infty$, Eq. (31), as expected for weak coupling. For the strong-coupling case $\left(z_{0}=0.1 \mathrm{c} / \omega_{p}\right)$ at $\omega_{0}=0.65 \omega_{p}, \alpha_{p}=0.282 \omega_{0}$, whereas the frequency-shift FM approximation gives $\delta_{g}=0.3198 \omega_{0}$, and

$$
|c|=\left|b_{g o}^{\text {Res }}(t)\right|=\frac{1}{\left|D^{\prime}\left(s_{p}\right)\right|}=0.893 \text {. }
$$

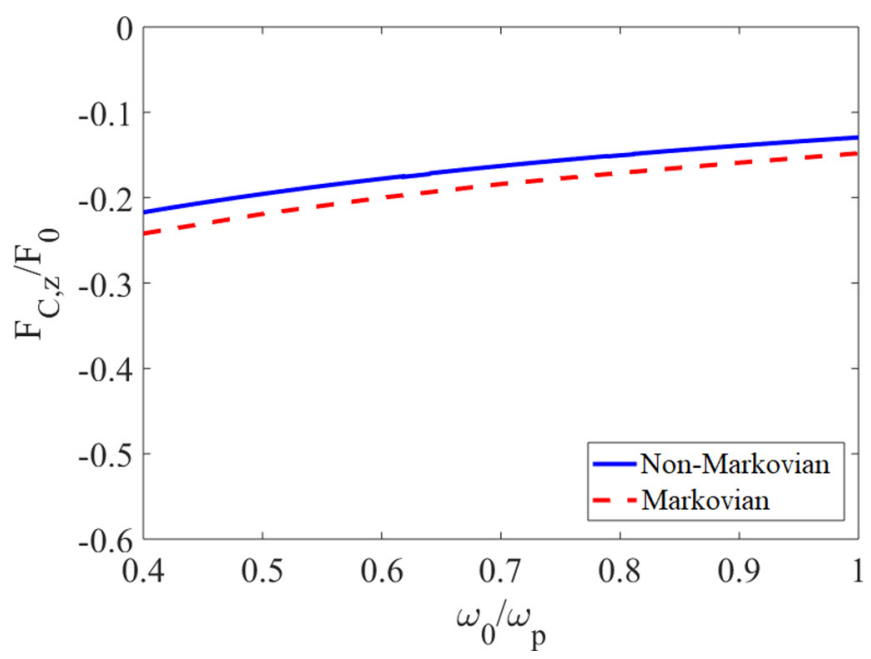

FIG. 10. Casimir force $(t \rightarrow \infty)$ comparing the Markov and non-Markovian results for the weak-coupling situation, $z_{0}=$ $0.7 c / \omega_{p}, g=0.0455$. 
As expected, $\alpha_{p} \neq \delta_{g}$ and $|c| \neq 1$ for the strongly nonMarkovian case.

Figure 10 shows the non-Markovian Casimir-Polder force $(t \rightarrow \infty)$ obtained from the residue leading to (30) and the FM approximation in the weak-coupling case. It can be seen that the agreement, and the trend, agree fairly well.

\section{CONCLUSIONS}

The non-Markovian time dynamics of two-level atoms immersed in inhomogeneous, nonreciprocal environments has been studied using Weisskopf-Wigner theory in the strongand weak-coupling regimes. Ground-state and excited atoms were considered as two separate initial-value problems. For atoms close to a material interface, strong coupling results in strongly non-Markovian behavior. Various approximations were also discussed, and the transient Casimir-Polder force was obtained.

Our analysis reveals that the standard Markovian-type formulas used to predict the instantaneous fluctuation-induced (Casimir-Polder) forces in atomic systems can be inaccurate, as they neglect transients where the force can switch sign and exhibit strong oscillations. This effect is especially important in the strong-coupling regime, where the usual theory totally breaks down. Furthermore, we have highlighted that the states $|e, 0\rangle$ and $|g, 0\rangle$ are projected onto orthogonal subspaces of the interacting light-matter system and thereby their time evolution is determined by two orthogonal bases of product states.

\section{ACKNOWLEDGMENTS}

The authors gratefully acknowledge discussions with Steve Hughes and Peter Milonni. This work was supported in part by (M. S.) Fundação para a Ciência e a Tecnologia and by Instituto de Telecomunicações under project UID/EEA/50008/2019.

\section{APPENDIX A: GREEN FUNCTION}

Although the treatment is fully quantum at a macroscopic level, the needed Green function is the classical Green function, arising from classical Maxwell's equations, and is provided in Refs. [9,10] (however, the notation for the Green function here differs from that used in $[9,10]$ by a factor of $\left.i \omega \mu_{0}\right)$. The Green function has vacuum and scattered contributions, where the vacuum term, divergent in the dipole approximation, leads to the Lamb shift. We assume that the Lamb shift is accounted for in the definition of the atomic transition frequency $\omega_{0}$, and in the following we use the scattered Green function, which dominates the material response for close atom-interface separations. The relationship between the electric field and the Green function is [29-35]

$$
\hat{\mathbf{E}}=i \omega \mu \int d^{3} \mathbf{r}^{\prime} \mathbf{G}\left(\mathbf{r}, \mathbf{r}^{\prime}, \omega\right) \cdot \mathbf{J}_{N}\left(\mathbf{r}^{\prime}, \omega\right)
$$

where

$$
\hat{\mathbf{J}}_{N}(\mathbf{r}, \omega)=\omega \sqrt{\frac{\hbar \varepsilon_{0}}{\pi}} \mathbf{T}(\mathbf{r}, \omega) \cdot \hat{\mathbf{f}}(\mathbf{r}, \omega)
$$

is the noise current, $\hat{\mathbf{f}}, \hat{\mathbf{f}}^{\dagger}$ are the canonically conjugate field variables, and where $\mathbf{T}\left(\mathbf{r}, \omega_{\lambda}\right) \cdot \mathbf{T}^{\dagger}\left(\mathbf{r}, \omega_{\lambda}\right)=$ $\frac{1}{2 i}\left[\varepsilon(\mathbf{r}, \omega)-\varepsilon^{\dagger}(\mathbf{r}, \omega)\right]$ accounts for the material environment.

The Green function satisfies [33]

$$
\begin{aligned}
2 i \frac{\omega^{2}}{c^{2}} \int & d^{3} \mathrm{r}^{\prime} K_{i k}\left(\mathbf{r}, \mathbf{r}^{\prime}, \omega_{\lambda}\right) K_{j k}^{*}\left(\mathbf{r}_{0}, \mathbf{r}^{\prime}, \omega_{\lambda}\right) \\
& =G_{i j}\left(\mathbf{r}, \mathbf{r}_{0}, \omega_{\lambda}\right)-G_{i j}^{*}\left(\mathbf{r}_{0}, \mathbf{r}, \omega_{\lambda}\right)=G_{\mathbf{I}, i, j}\left(\mathbf{r}, \mathbf{r}_{0}, \omega_{\lambda}\right) .
\end{aligned}
$$

Writing a scalar component of the Green function as

$$
\begin{aligned}
G\left(\mathbf{r}, \mathbf{r}_{0}, \omega\right) \sim & \int d k_{x} d k_{y}\left(\widetilde{G}_{r}\left(k_{x}, k_{y}\right)+i \widetilde{G}_{i}\left(k_{x}, k_{y}\right)\right) \\
& \times e^{i k_{x}\left(x-x_{0}\right)} e^{i k_{y}\left(y-y_{0}\right)} e^{-\gamma_{0}\left(k_{x}, k_{y}\right)\left(z+z_{0}\right)},
\end{aligned}
$$

for the layered environment depicted in Fig. 1, where $\gamma_{0}=$ $\sqrt{k_{x}^{2}+k_{y}^{2}-k_{0}^{2}}$, it is easily shown that

$$
\begin{aligned}
\frac{\partial}{\partial \alpha} & \frac{\gamma \cdot \mathbf{G}_{\mathbf{I}}\left(\mathbf{r}, \mathbf{r}_{0}, \omega\right) \cdot \gamma}{2 i} \\
& =\left\{\begin{aligned}
-i \operatorname{Re} \frac{\partial}{\partial \alpha} \gamma \cdot \mathbf{G}\left(\mathbf{r}_{0}, \mathbf{r}_{0}, \omega\right) \cdot \gamma, & \alpha=x, y \\
\operatorname{Im} \frac{\partial}{\partial \alpha} \gamma \cdot \mathbf{G}\left(\mathbf{r}_{0}, \mathbf{r}_{0}, \omega\right) \cdot \gamma, & \alpha=z .
\end{aligned}\right.
\end{aligned}
$$

\section{APPENDIX B: NUMERICAL SOLUTION OF VOLTERRA INTEGRAL EQUATION}

In order to numerically solve the Volterra integral equation (10) having the form

$$
\frac{d}{d t} c(t)=\int_{0}^{t} H\left(t, t^{\prime}\right) c\left(t^{\prime}\right) d t^{\prime},
$$

a grid can be defined [43] $t_{i}=0+i h, \quad i=0,1, \ldots N$, where $h=t_{\text {final }} / N$ with $N$ the number of grid points $\left(t_{\text {initial }}=0\right.$ is implicit), and using a trapezoidal rule

$$
\begin{aligned}
& \int_{0}^{t_{i}} H\left(t_{i}, t^{\prime}\right) c\left(t^{\prime}\right) d t^{\prime} \\
& \quad=h\left(\frac{1}{2} H_{i 0} c_{0}+\sum_{j=1}^{i-1} H_{i j} c_{j}+\frac{1}{2} H_{i i} c_{i}\right),
\end{aligned}
$$

where $H_{i j}=H\left(t_{i}, t_{j}\right), c_{j}=c\left(t_{j}\right)$. Writing the derivative as

$$
\frac{d}{d t} c(t)=\frac{c(t+h)-c(t)}{h},
$$

then

$$
\frac{c_{(i+1)}-c_{i}}{h}-h \frac{1}{2} H_{i i} c_{i}=h\left(\frac{1}{2} H_{i 0} c_{0}+\sum_{j=1}^{i-1} H_{i j} c_{j}\right),
$$

$i=0,1,2, \ldots N$, where for $i=0, c_{0}=1$. In general,

$$
\begin{aligned}
c_{m}= & \left(1+h^{2} \frac{1}{2} H_{(m-1)(m-1)}\right) c_{(m-1)} \\
& +h^{2}\left(\frac{1}{2} H_{(m-1) 0}+\sum_{j=1}^{m-2} H_{(m-1) j} c_{j}\right), \quad m=1,2, \ldots N .
\end{aligned}
$$




\section{APPENDIX C: MARKOV APPROXIMATIONS OF THE POPULATION}

\section{Excited atom}

Various Markov-type approximations can be made in evaluating the time integral in (10) for the weak-coupling case, where the result is essentially Markovian. The first approximation is to assume that the population has no memory (Markov approximation, MA), $c_{e o}\left(t^{\prime}\right) \simeq c_{e o}(t)$, and the second approximation is to extend the upper limit of the integration to infinity, which can typically be justified by noting that the most important contribution to the integral comes from the vicinity of $\omega_{\lambda}=\omega_{0}$. Then, the Sokhotski-Plemelj (SP) identity,

$$
\begin{aligned}
& \int_{0}^{t} e^{ \pm i\left(\omega-\omega_{0}\right)\left(t-t^{\prime}\right)} d t^{\prime} \\
& \quad \rightarrow \int_{0}^{\infty} e^{ \pm i\left(\omega-\omega_{0}\right)\left(t-t^{\prime}\right)} d t^{\prime}=\pi \delta\left(\omega-\omega_{0}\right) \pm i \operatorname{PV}\left(\frac{1}{\omega-\omega_{0}}\right),
\end{aligned}
$$

leads to the usual resonant and nonresonant contributions. Since these two approximations are often used together, we will refer to this as the full Markov (FM) approximation.

Another option is to assume that the population has no memory (MA) but that the upper limit of the integration is not extended to infinity, leading to

$$
\int_{0}^{t} e^{-i\left(\omega-\omega_{0}\right)\left(t-t^{\prime}\right)} d t^{\prime}=\frac{1-e^{-i\left(\omega-\omega_{0}\right) t}}{i\left(\omega-\omega_{0}\right)} .
$$

We refer to this as the partial Markov (PM) approximation. In the following it will be useful to refer to the function

$$
h_{e}(\mathbf{r}, \mathbf{r}, \omega, g)=\frac{1}{\hbar \pi \varepsilon_{0}} \int_{0}^{\infty} d \omega_{\lambda} \frac{\omega_{\lambda}^{2}}{c^{2}} \frac{\gamma \cdot \mathbf{G}_{\mathbf{I}}\left(\mathbf{r}, \mathbf{r}, \omega_{\lambda}\right) \cdot \gamma}{2 i\left(\omega_{\lambda}-\omega\right)} g,
$$

where $g=g\left(\omega_{\lambda}, t\right)$.

The FM approximation of the Volterra integral equation yields

$$
\frac{d}{d t} c_{e o}^{\mathrm{FM}}(t)=\left(-\Gamma^{\mathrm{FM}} \frac{1}{2}+i \delta^{\mathrm{FM}}\right) c_{e o}^{\mathrm{FM}}(t),
$$

with an energy shift $\delta^{\mathrm{FM}}=\delta=\mathrm{PV}\left[h_{e}\left(\mathbf{r}_{\mathbf{0}}, \mathbf{r}_{\mathbf{0}}, \omega_{0}, 1\right)\right]$, where $\mathrm{PV}$ indicates a principal-value integral, and a decay rate $\Gamma^{\mathrm{FM}}$ of

$$
\Gamma^{\mathrm{FM}}=\Gamma=\frac{2 \omega_{0}^{2}}{\hbar \varepsilon_{0} c^{2}} \frac{\gamma \cdot \mathbf{G}_{\mathbf{I}}\left(\mathbf{r}_{0}, \mathbf{r}_{0}, \omega_{0}\right) \cdot \gamma}{2 i} .
$$

Since $\gamma \cdot \mathbf{G}_{\mathbf{I}}\left(\mathbf{r}_{0}, \mathbf{r}_{0}, \omega_{0}\right) \cdot \gamma=2 i \operatorname{Im} \gamma \cdot \mathbf{G}\left(\mathbf{r}_{0}, \mathbf{r}_{0}, \omega_{0}\right) \cdot \gamma$ for a linear dipole, $\Gamma$ and $\delta$ are seen to be real-valued, as required, and provide the usual exponential decay and energy shift of $-\hbar \delta$, which agrees with the well-known expressions for reciprocal media [22]. Therefore, for a linear dipole the form of $\Gamma$ and $\delta$ in terms of the Green function are the same in the reciprocal and nonreciprocal case.

From $(\mathrm{C} 4), c_{e o}^{\mathrm{FM}}(t)=c_{e o}^{\mathrm{FM}}(0) e^{-\Gamma \frac{1}{2} t} e^{i \delta t}$, such that the FM amplitude of the state $|e, 0\rangle$ is

$$
b_{e o}^{\mathrm{FM}}(t)=c_{e o}^{\mathrm{FM}}(0) e^{-\Gamma \frac{1}{2} t} e^{-i\left(\omega_{0}-\delta\right) t},
$$

with $c_{e o}^{\mathrm{FM}}(0)=1$ by assumption of the initial-value condition.
In the PM approximation,

$$
\frac{d}{d t} c_{e o}^{\mathrm{PM}}(t) \simeq-c_{e o}^{\mathrm{PM}}(t) p_{e}(t),
$$

where $\quad p_{e}(t)=\mathrm{PV}\left[h_{e}\left(\mathbf{r}_{0}, \mathbf{r}_{0}, \omega_{0}, f_{e}(t)\right)\right], \quad f_{e}(t)=$ $-i\left(1-e^{-i\left(\omega_{\lambda}-\omega_{0}\right) t}\right)$. The solution of $(\mathrm{C} 7)$ is

$$
c_{e o}^{\mathrm{PM}}(t)=e^{i h_{e} t} e^{q_{e}(t)-q_{e}(0)},
$$

where $q_{e}(t)=\mathrm{PV}\left[h_{e}\left(\mathbf{r}_{0}, \mathbf{r}_{0}, \omega_{0}, g_{e}(t)\right)\right], g_{e}(t)=e^{-i\left(\omega_{\lambda}-\omega_{0}\right) t} /$ $\left(\omega_{\lambda}-\omega_{0}\right)$.

Since by causality $\mathbf{G}_{\mathbf{I}}\left(\mathbf{r}_{0}, \mathbf{r}_{0}, \omega_{\lambda}\right)$ must be analytic in the upper-half $\omega_{\lambda}$ plane and $\lim _{\left|\omega_{\lambda}\right| \rightarrow \infty}\left(\omega_{\lambda}^{2} / c^{2}\right) \mathbf{G}_{\mathbf{I}}\left(\mathbf{r}, \mathbf{r}_{0}, \omega_{\lambda}\right)=0$, the integral for $h_{e}$ can be closed with a semicircle in the first quadrant of the complex $\omega_{\lambda}$ plane, resulting in an integral over positive imaginary frequencies.

\section{Ground-state atom}

For the ground-state atom, in the PM approximation of (17)

$$
\frac{d}{d t} b_{g o}^{\mathrm{PM}}(t)=-b_{g o}^{\mathrm{PM}}(t) p_{g}(t),
$$

where $\quad p_{g}(t)=h_{e}\left(\mathbf{r}_{0}, \mathbf{r}_{0},-\omega_{0}, f_{g}(t)\right), f_{g}(t)=$ $-i\left(1-e^{-i\left(\omega_{\lambda}+\omega_{0}\right)} t\right)$. The solution of (C9) is

$$
b_{g o}^{\mathrm{PM}}(t)=e^{i \delta_{g} t} e^{q_{g}(t)-q_{g}(0)},
$$

where $\delta_{g}=h_{e}\left(\mathbf{r}_{0}, \mathbf{r}_{0},-\omega_{0}, 1\right), q_{g}(t)=h_{e}\left(\mathbf{r}_{0}, \mathbf{r}_{0},-\omega_{0}, r_{g}(t)\right)$, and $r_{g}(t)=e^{-i\left(\omega_{\lambda}+\omega_{0}\right) t} /\left(\omega_{\lambda}+\omega_{0}\right)$.

It can be seen that $q_{g}(t)$ rapidly becomes small as $t$ increases due to the rapidly oscillating integrand, and so

$$
b_{g o}^{\mathrm{PM}}(t) \simeq e^{i \delta_{g} t},
$$

which agrees with the result from the full Markov approximation, $\frac{d}{d t} b_{g o}^{\mathrm{FM}}(t)=b_{g o}^{\mathrm{FM}}(t) i \delta_{g}$, so that

$$
b_{g o}^{\mathrm{FM}}(t)=e^{i \delta_{g} t} .
$$

Therefore, in the Markov approximation, the state $|g, 0\rangle$ has no decay [40], unlike the state $|e, 0\rangle$. The relative energy difference between the states $|e, 1\rangle$ and $|g, 0\rangle$ is $\hbar \omega_{0}$.

\section{APPENDIX D: MARKOV APPROXIMATIONS OF THE FORCE}

\section{Excited atom}

The exact, generally non-Markovian force is given by (15). There are several combinations of Markov-type approximations that can be used to approximate the force in the weakcoupling case. One form of Markov approximation of the force is obtained by substituting the PM or FM approximations for the population into the force expression and then evaluating the resulting time integral exactly. In this manner, for example, the resulting FM approximation of the force is

$$
\begin{aligned}
\mathscr{F}_{\alpha}^{\mathrm{FM}}(t) \\
=2 \operatorname{Re}\left\{\left.\frac{i}{\pi \varepsilon_{0}} \int_{0}^{\infty} d \omega_{\lambda} \frac{\omega_{\lambda}^{2}}{c^{2}} \frac{\partial}{\partial \alpha} \frac{\gamma \cdot \mathbf{G}_{\mathbf{I}}\left(\mathbf{r}, \mathbf{r}_{0}, \omega_{\lambda}\right) \cdot \gamma}{2 i}\right|_{\mathbf{r}=\mathbf{r}_{0}}\right. \\
\left.\quad \times e^{-\Gamma \frac{1}{2} t} \frac{e^{-i\left(\omega_{\lambda}-\omega_{0}+\delta\right) t}-e^{-\Gamma \frac{1}{2} t}}{\Gamma-i\left(\omega_{\lambda}-\omega_{0}+\delta\right)}\right\}
\end{aligned}
$$


and similarly for $\mathscr{F}_{\alpha}^{\mathrm{PM}}$. Alternatively, one could first impose the Markov approximation $c_{e o}\left(t^{\prime}\right) \simeq c_{e o}(t)$ directly in the time integral in (15), then evaluate the resulting time integral exactly, resulting in

$$
\begin{aligned}
\mathscr{F}_{\alpha}^{\mathrm{PM} 2}(t) \simeq & \frac{2}{\pi \varepsilon_{0}}\left|c_{e o}(t)\right|^{2} \\
& \times \operatorname{Re}\left\{\left.\int_{0}^{\infty} d \omega_{\lambda} \frac{\omega_{\lambda}^{2}}{c^{2}} \frac{\partial}{\partial \alpha} \frac{\gamma \cdot \mathbf{G}_{\mathbf{I}}\left(\mathbf{r}, \mathbf{r}_{0}, \omega_{\lambda}\right) \cdot \gamma}{2 i}\right|_{\mathbf{r}=\mathbf{r}_{0}}\right. \\
& \left.\times \frac{1-e^{-i\left(\omega_{\lambda}-\omega_{0}\right) t}}{\left(\omega_{\lambda}-\omega_{0}\right)}\right\} .
\end{aligned}
$$

As a further approximation, the upper limit of the time integral could be extended to $t \rightarrow \infty$, allowing the SP identity to be used. However, this leads to nonzero force at $t=0$.

In a Markovian approximation, the Casimir-Polder force can be obtained as a derivative of the Markovian energy shift, $\hbar \delta^{\mathrm{FM}}=\hbar \mathrm{PV}\left[h_{e}\left(\mathbf{r}, \mathbf{r}, \omega_{0}, 1\right)\right]$, which is the same as [[15], (4.39), (6.77)]. There they assume an excited atom, akin to starting with the state $|e, 0\rangle$. The $\mathrm{CP}$ force can then be written as the total differential of the energy shift,

$$
\begin{aligned}
\mathscr{F} \mathscr{C}_{z} & =-d(-\hbar \delta(\mathbf{r})) \\
& =\left.\frac{2}{\pi \varepsilon_{0}} \operatorname{PV} \int_{0}^{\infty} d \omega_{\lambda} \frac{\omega_{\lambda}^{2}}{c^{2}} \frac{\partial}{\partial z} \frac{\gamma \cdot \mathbf{G}_{\mathbf{I}}\left(\mathbf{r}, \mathbf{r}_{0}, \omega_{\lambda}\right) \cdot \gamma}{2 i\left(\omega_{\lambda}-\omega_{0}\right)}\right|_{\mathbf{r}=\mathbf{r}_{0}} .
\end{aligned}
$$

Using the Wick rotation, complex-plane analysis leads to resonant and nonresonant components.

\section{Ground-state atom}

For the force on a ground-state atom, Eq. (19), if one first evaluates the PM or FM population, $b_{g o}^{\mathrm{FM}}(t)=e^{i \delta_{g} t} \simeq b_{g o}^{\mathrm{PM}}(t)$, and inserts this into the force equation and evaluates the time integral exactly, the result is, e.g.,

$$
\begin{aligned}
\mathscr{F}_{\alpha}^{\mathrm{FM}}(t)= & 2 \operatorname{Re}\left\{\frac{i}{\pi \varepsilon_{0}} \int_{0}^{\infty} d \omega_{\lambda} \frac{\omega_{\lambda}^{2}}{c^{2}} \frac{\partial}{\partial \alpha} \frac{\gamma \cdot \mathbf{G}_{\mathbf{I}}\left(\mathbf{r}, \mathbf{r}_{0}, \omega_{\lambda}\right) \cdot \gamma}{2 i}\right. \\
& \left.\times \frac{1-e^{-i\left(\omega_{\lambda}+\omega_{0}+\delta_{g}\right) t}}{\omega_{\lambda}+\omega_{0}+\delta_{g}}\right\} .
\end{aligned}
$$

An other option is to first impose the Markov approximation $b_{g o}\left(t^{\prime}\right) \simeq b_{g o}(t)$ in the time integral in (19) and then evaluate the time integral exactly, without extending the upper limit to $t \rightarrow \infty$ (PM2), or extending the upper limit to $t \rightarrow \infty$ and using the SP identity (FM2), leading to

$$
\begin{aligned}
\mathscr{F}_{\alpha}^{\mathrm{PM} 2}(t) \simeq & \left|b_{g o}(t)\right|^{2} \frac{2}{\pi \varepsilon_{0}} \\
& \times\left\{\left.\operatorname{Re} \int_{0}^{\infty} d \omega_{\lambda} \frac{\omega_{\lambda}^{2}}{c^{2}} \frac{\partial}{\partial \alpha} \frac{\gamma \cdot \mathbf{G}_{\mathbf{I}}\left(\mathbf{r}, \mathbf{r}_{0}, \omega_{\lambda}\right) \cdot \gamma}{2 i}\right|_{\mathbf{r}=\mathbf{r}_{0}}\right. \\
& \left.\times \frac{1-e^{-i\left(\omega_{\lambda}+\omega_{0}\right) t}}{\omega_{\lambda}+\omega_{0}}\right\}, \\
= & \left|b_{g o}(t)\right|^{2}\left(\mathscr{F}_{\alpha, \text { static }}^{\mathrm{PM} 2}+\mathscr{F}_{\alpha, \text { dynamic }}^{\mathrm{PM} 2}(t)\right) \\
\simeq & \mathscr{F}_{\alpha, \text { static }}^{\mathrm{PM} 2}+\mathscr{F}_{\alpha, \text { dynamic }}^{\mathrm{PM} 2}(t) \\
\simeq & \left|b_{g o}(t)\right|^{2} \frac{2}{\pi \varepsilon_{0}} \quad \text { (D5) } \\
\mathscr{F}_{\alpha}^{\mathrm{FM} 2}(t) & \times \operatorname{Re}\left\{\left.\int_{0}^{\infty} d \omega_{\lambda} \frac{\omega_{\lambda}^{2}}{c^{2}} \frac{\partial}{\partial \alpha} \frac{\gamma \cdot \mathbf{G}_{\mathbf{I}}\left(\mathbf{r}, \mathbf{r}_{0}, \omega_{\lambda}\right) \cdot \gamma}{2 i\left(\omega_{\lambda}+\omega_{0}\right)}\right|_{\mathbf{r}=\mathbf{r}_{0}}\right\} \\
= & \left|b_{g o}(t)\right|^{2} \mathscr{F}_{\alpha, \text { static }}^{\mathrm{PM} 2} \simeq \mathscr{F}_{\alpha, \text { static }}^{\mathrm{PM} 2},
\end{aligned}
$$

where $\left|b_{g o}(t)\right|^{2} \simeq 1$ for weak coupling. Since $\delta_{g} \ll \omega_{0}$, $\mathscr{F}_{\alpha}^{\mathrm{PM}}(t) \simeq \mathscr{F}_{\alpha}^{\mathrm{PM} 2}(t)$. The static and dynamic terms $\mathscr{F}_{\alpha \text {, static }}^{\mathrm{PM} 2}$ and $\mathscr{F}_{\alpha, \text { dynamic }}^{\mathrm{PM} 2}(t)$ correspond to static and dynamic potentials that agree with [[19], (15)].

The FM approximation (D4) and PM2 approximation (D5) agree very well with the exact force (19) for the weakcoupling case, since the system is essentially Markovian. The FM2 approximation results in $\mathscr{F}_{\alpha}^{\mathrm{FM} 2}(0) \neq 0$, but for longer times, $\mathscr{F}_{\alpha}^{\mathrm{FM}}(t) \simeq \mathscr{F}_{\alpha}^{\mathrm{PM}}(t) \simeq \mathscr{F}_{\alpha}^{\mathrm{FM} 2}(t) \simeq \mathscr{F}_{\alpha}(t)$. Since this is the force on the ground-state atom, this can be considered as the CP force, $\mathscr{F} C_{\alpha}$.

For the Casimir-Polder force, from the FM approximation of the population, $\hbar \delta_{g}=\hbar h_{e}\left(\mathbf{r}, \mathbf{r},-\omega_{0}, 1\right)$, which is the same as [[14], (4.50)]. (There they assume a ground-state atom, which is essentially the same as starting with the state $|g, 0\rangle$.) Then we can write the Casimir-Polder force as the total differential of the energy shift, $\mathscr{F} \mathscr{C}=-d\left(-\hbar \delta_{g}(\mathbf{r})\right)$, which is the same as (D6).
[1] S. Chu, Nobel lecture: The manipulation of neutral particles, Rev. Mod. Phys. 70, 685 (1998).

[2] W. D. Phillips, Nobel lecture: Laser cooling and trapping of neutral atoms, Rev. Mod. Phys. 70, 721 (1998).

[3] C. N. Cohen-Tannoudji, Nobel lecture: Manipulating atoms with photons, Rev. Mod. Phys. 70, 707 (1998).

[4] M. Saffman, T. G. Walker, and K. Mølmer, Quantum information with Rydberg atoms, Rev. Mod. Phys. 82, 2313 (2010).

[5] H. B. G. Casimir and D. Polder, The influence of retardation on the London-van der Waals forces, Phys. Rev. 73, 360 (1948).
[6] I. E. Dzyaloshinskii, E. M. Lifshitz, and L. P. Pitaevskii, The general theory of van der Waals forces, Adv. Phys. 38, 165 (1961)

[7] F. Intravaia, C. Henkel, and M. Antezza, Fluctuation-induced forces between atoms and surfaces: The Casimir-Polder interaction, in Casimir Physics, edited by D. Dalvit, P. Milonni, D. Roberts, and F. da Rosa, Lecture Notes in Physics Vol. 834 (Springer-Verlag, Berlin/Heidelberg, 2011), Chap. 11, p. 345.

[8] M. G. Silveirinha, S. A. Hassani Gangaraj, G. W. Hanson, and M. Antezza, Fluctuation-induced forces on an atom near a photonic topological material, Phys. Rev. A 97, 022509 (2018). 
[9] S. A. H. Gangaraj, G. W. Hanson, M. Antezza, and M. G. Silveirinha, Spontaneous lateral atomic recoil force close to a photonic topological material, Phys. Rev. B 97, 201108(R) (2018).

[10] S. A. H. Gangaraj, M. G. Silveirinha, G. W. Hanson, M. Antezza, and F. Monticone, Quantum optical torque on a twolevel system near a photonic topological material, Phys. Rev. B 98, 125146 (2018).

[11] S. Y. Buhmann, L. Knoll, D.-G. Welsch, H. T. Dung, CasimirPolder forces: A nonperturbative approach, Phys. Rev. A 70, 052117 (2004).

[12] S. Y. Buhmann and S. Scheel, Thermal Casimir Versus CasimirPolder Forces: Equilibrium and Nonequilibrium Forces, Phys. Rev. Lett. 100, 253201 (2008).

[13] G. Compagno, G. M. Palma, R. Passante, and F. Persico, Atoms dressed and partially dressed by the zeropoint fluctuations of the electromagnetic fields, J. Phys. B: At. Mol. Opt. Phys. 28, 1105 (1995).

[14] S. Y. Buhmann, Dispersion Forces I, Springer Tracts in Modern Physics Vol. 247 (Springer Nature, Cham, Switzerland, 2012).

[15] S. Y. Buhmann, Dispersion Forces II, Springer Tracts in Modern Physics, Vol. 248 (Springer Nature, Cham, Switzerland, 2012).

[16] F. Intravaia, R. O. Behunin, C. Henkel, K. Busch, and D. A. R. Dalvit, Non-Markovianity in atom-surface dispersion forces, Phys. Rev. A 94, 042114 (2016).

[17] V. V. Dodonov, Current status of the dynamical Casimir effect, Phys. Scr. 82, 038105 (2010).

[18] R. Vasile and R. Passante, Dynamical Casimir-Polder force between an atom and a conducting wall, Phys. Rev. A 78, 032108 (2008).

[19] H. R. Haakh, C. Henkel, S. Spagnolo, L. Rizzuto, and R. Passante, Dynamical Casimir-Polder interaction between an atom and surface plasmons, Phys. Rev. A 89, 022509 (2014).

[20] P. Yao, C. Van Vlack, A. Reza, M. Patterson, M. M. Dignam, and S. Hughes, Ultrahigh Purcell factors and Lamb shifts in slow-light metamaterial waveguides, Phys. Rev. B 80, 195106 (2009).

[21] V. Weisskopf and E. Wigner, Berechnung der natürlichen linienbreite auf grund der diracschen lichttheorie (Calculation of the natural line width on the basis of Dirac's theory of light), Zeitschrift für Physik, 63, 54 (1930), Translated by J. B. Sykes and reprinted in W. Hindmarsh, Atomic Spectra (Pergamon Press, Oxford/New York, 1967), pp. 304-327.

[22] W. Vogel and D.-G. Welsch, Quantum Optics (Wiley-VCH, Weinheim, Germany, 2006).

[23] P. Lodah, S. Mahmoodian, and S. Stobbe, Interfacing single photons and single quantum dots with photonic nanostructures, Rev. Mod. Phys. 87, 347 (2015).

[24] K. H. Madsen, S. Ates, T. Lund-Hansen, A. Löffler, S. Reitzenstein, A. Forchel, and P. Lodah, Observation of NonMarkovian Dynamics of a Single Quantum Dot in a Micropillar, Phys. Rev. Lett. 106, 233601 (2011).

[25] C.-J. Yang and J.-H. Am, Suppressed dissipation of a quantum emitter coupled to surface plasmon polaritons, Phys. Rev. B 95, 161408(R) (2017).
[26] E. Palik, R. Kaplan, R. Gammon, H. Kaplan, R. Wallis, and J. Quinn, Coupled surface magnetoplasmon-optic-phonon polariton modes on InSb, Phys. Rev. B 13, 2497 (1976).

[27] J. A. Bittencourt, Fundamentals of Plasma Physics, 3rd ed. (Springer-Verlag, New York, 2010).

[28] L. Knöll, S. Scheel, and D.-G. Welsch, QED in dispersing and absorbing media, in Coherence and Statistics of Photons and Atoms, edited by J. Perina (Wiley-VCH, Weinheim, Germany, 2001).

[29] T. Gruner and D.-G. Welsch, Green-function approach to the radiation-field quantization for homogeneous and inhomogeneous Kramers-Kronig dielectrics, Phys. Rev. A 53, 1818 (1996).

[30] H. T. Dung, L. Knöll, and D.-G. Welsch, Three-dimensional quantization of the electromagnetic field in dispersive and absorbing inhomogeneous dielectrics, Phys. Rev. A 57, 3931 (1998).

[31] H. T. Dung, L. Knöll, and D.-G. Welsch, Spontaneous decay in the presence of dispersing and absorbing bodies: General theory and application to a spherical cavity, Phys. Rev. A 62, 053804 (2000).

[32] S. Y. Buhmann, D. T. Butcher, and S. Scheel, Macroscopic quantum electrodynamics in nonlocal and nonreciprocal media, New. J. Phys. 114, 083034 (2012).

[33] S. A. Hassani Gangaraj, G. W. Hanson, and M. Antezza, Robust entanglement with three-dimensional nonreciprocal photonic topological insulators, Phys. Rev. A 95, 063807 (2017).

[34] A. Drezet, Equivalence between the Hamiltonian and Langevin noise descriptions of plasmon polaritons in a dispersive and lossy inhomogeneous medium, Phys. Rev. A 96, 033849 (2017).

[35] T. G. Philbin, Canonical quantization of macroscopic electromagnetism, New J. Phys 12, 123008 (2010).

[36] M. Wubs, Transient QED effects in absorbing dielectrics, Phys. Rev. A 63, 043809 (2001).

[37] L. Fonda, G. C. Ghirardi, and A. Rimini, Decay theory of unstable quantum systems, Rep. Prog. Phys. 141, 587 (1978).

[38] P. R. Berman and G. W. Ford, Spontaneous decay, unitarity, and the Weisskopf-Wigner approximation, Adv. At., Mol., Opt. Phys. 59, 175 (2010).

[39] P. R. Berman and G. W. Ford, Spectrum in spontaneous emission: Beyond the Weisskopf-Wigner approximation, Phys. Rev. A 82, 023818 (2010).

[40] P. Milonni, The Quantum Vacuum (Academic Press, New York, 1994).

[41] J. P. Gordon and A. Ashkin, Motion of atoms in a radiation trap, Phys. Rev. A 21, 1606 (1980).

[42] The force $\mathscr{F}_{\alpha}(0)=0$ is expected based on the following argument: In the Heisenberg picture all the operators at $t=0$ are taken as the free-field operators, and the initial state is by assumption the product of the ground states of the noninteracting Hamiltonians. Therefore, $\left\langle\hat{\mathbf{p}}(0) \cdot \partial_{\alpha} \hat{\mathbf{E}}(\mathbf{r}, 0)\right\rangle=\langle\hat{\mathbf{p}}(0)\rangle$. $\left\langle\partial_{\alpha} \hat{\mathbf{E}}(\mathbf{r}, 0)\right\rangle=0 \cdot 0=0$ for all components of the force.

[43] W. H. Press, S. A. Teukolsky, W. T. Vetterling, and B. P. Flannery, Numerical Recipes (Cambridge University Press, Cambridge, England, 2007). 